\title{
Property of Tensor Satisfying Binary Law 2
}

\section{Koji Ichidayama}

Okayama, Japan

Email: ichikoji@lime.ocn.ne.jp

How to cite this paper: Ichidayama, K. (2020) Property of Tensor Satisfying Binary Law 2. Journal of Modern Physics, 11, 1649-1671.

https://doi.org/10.4236/jmp.2020.1110103

Received: September 13, 2020

Accepted: October 23, 2020

Published: October 26, 2020

Copyright () 2020 by author(s) and Scientific Research Publishing Inc. This work is licensed under the Creative Commons Attribution International License (CC BY 4.0).

http://creativecommons.org/licenses/by/4.0/

\begin{abstract}
I have already reported "Property of Tensor Satisfying Binary Law". This article is the article that I revise the contents of "Property of Tensor Satisfying Binary Law", and increase the report about new characteristics. We may arrive at the deeper understanding in this about "Property of Tensor Satisfying Binary Law".
\end{abstract}

\section{Keywords}

Tensor Covariant Derivative

\section{Introduction}

I have already reported "Property of Tensor Satisfying Binary Law" [1]. This article is the article that I revise the contents of "Property of Tensor Satisfying Binary Law", and increase the report about new characteristics. I show below it about the proposition supporting each for shifts from "Property of Tensor Satisfying Binary Law" to "[Property of Tensor Satisfying Binary Law 2]".

Proposition $1 \rightarrow$ [Proposition 2], Proposition $3 \rightarrow$ [Proposition 6], Proposition $4 \rightarrow$ [Proposition 7, Proposition 15], Proposition $5 \rightarrow$ [Proposition 3].

\section{Definition}

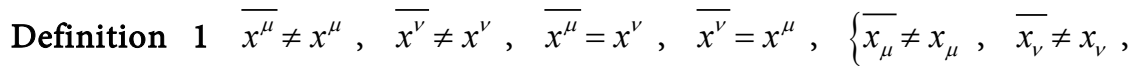
$\left.\overline{x_{\mu}}=x_{v}, \overline{x_{v}}=x_{\mu}\right\}$ is established [2]. I named $\overline{x^{\mu}} \neq x^{\mu}, \overline{x^{v}} \neq x^{v}, \overline{x^{\mu}}=x^{v}$, $\overline{x^{v}}=x^{\mu},\left\{\overline{x_{\mu}} \neq x_{\mu}, \overline{x_{v}} \neq x_{v}, \overline{x_{\mu}}=x_{v}, \overline{x_{v}}=x_{\mu}\right\} \quad$ "Binary Law" [2].

$\left\{\overline{x_{\mu}} \neq x_{\mu}, \overline{x_{v}} \neq x_{v}, \overline{x_{\mu}}=x_{v}, \overline{x_{v}}=x_{\mu}\right\}$ expresses a covariant form of Binary Law.

Definition 2 If $\overline{x^{\mu}} \neq x^{\mu}, \overline{x^{v}} \neq x^{\nu}, \overline{x^{\mu}}=x^{\nu}, \overline{x^{v}}=x^{\mu}$ is established, $x_{v}=x^{\mu}$ is established [2].

Definition 3 If $\overline{x^{\mu}} \neq x^{\mu}, \overline{x^{v}} \neq x^{\nu}, \overline{x^{\mu}}=x^{v}, \overline{x^{v}}=x^{\mu}$ is established, $x_{\mu}=x^{v}$ is established [2]. 
Definition 4 If $\overline{x^{\mu}} \neq x^{\mu}, \overline{x^{v}} \neq x^{v}, \overline{x^{\mu}}=x^{v}, \overline{x^{v}}=x^{\mu}$ is established, $\quad x_{v}=-x_{\mu}$ is established [2].

Definition 5 If $\overline{x^{\mu}} \neq x^{\mu}, \overline{x^{v}} \neq x^{v}, \overline{x^{\mu}}=x^{v}, \overline{x^{v}}=x^{\mu}$ is established, $x^{v}=-x^{\mu}$ is established [2].

Definition 6 If all coordinate systems $x^{\mu}, x^{\nu}, x^{\sigma}, x^{\lambda}, \cdots$ satisfy $\overline{x^{\mu}} \neq x^{\mu}$, $\overline{x^{v}} \neq x^{\nu}, \overline{x^{\mu}}=x^{\nu}, \overline{x^{\nu}}=x^{\mu}$, all coordinate systems $x^{\mu}, x^{v}, x^{\sigma}, x^{\lambda}, \cdots$ shifts to only two of $x^{\mu}, x^{\nu} \quad[2]$.

Definition $7 g_{\mu}^{\mu}=1, g_{v}^{\mu}=0:(\mu \neq v)$ is establishment [3].

Definition $8 \frac{\partial^{3} x^{\mu}}{\partial x^{v} \partial x^{v} \partial x^{v}}=M$ is established for $\frac{\partial^{3} x^{\mu}}{\partial x^{v} \partial x^{v} \partial x^{v}}$.

Definition $9 m_{; v}=\frac{\partial m}{\partial x^{v}}=0$ is established. " $m$ ” expresses Mass.

Hypothesis $1 m \propto M, m=\epsilon M$ is established. “ $M$ ” ' expresses $\frac{\partial^{3} x^{\mu}}{\partial x^{v} \partial x^{v} \partial x^{v}}=M$, “ $\epsilon$ ” expresses Proportional constant, and " $m$ " expresses Mass.

Definition 10 The first-order covariant derivative of the covariant vector satisfied $x_{\mu ; \nu}=\frac{\partial x_{\mu}}{\partial x^{v}}-x_{\tau} \Gamma_{\mu \nu}^{\tau}=\frac{\partial x_{\mu}}{\partial x^{v}}-x_{\tau} \frac{1}{2} g^{\epsilon \tau}\left(\frac{\partial g_{\mu \epsilon}}{\partial x^{v}}+\frac{\partial g_{v \epsilon}}{\partial x^{\mu}}-\frac{\partial g_{\mu v}}{\partial x^{\epsilon}}\right)$ [4].

Definition 11 The first-order covariant derivative of the contravariant vector satisfied $x_{; \nu}^{\mu}=\frac{\partial x^{\mu}}{\partial x^{v}}+x^{\tau} \Gamma_{\tau v}^{\mu}=\frac{\partial x^{\mu}}{\partial x^{v}}+x^{\tau} \frac{1}{2} g^{\epsilon \mu}\left(\frac{\partial g_{\tau \epsilon}}{\partial x^{v}}+\frac{\partial g_{v \epsilon}}{\partial x^{\tau}}-\frac{\partial g_{\tau v}}{\partial x^{\epsilon}}\right)$ [4].

Definition 12 The second-order covariant derivative of the contravariant vector satisfied

$$
\begin{aligned}
& x_{;, \sigma \sigma}^{\mu}=\frac{\partial x_{; \nu}^{\mu}}{\partial x^{\sigma}}+x_{; \nu}^{l} \Gamma_{l \sigma}^{\mu}-x_{; l}^{\mu} \Gamma_{v \sigma}^{t} \\
& =\frac{\partial}{\partial x^{\sigma}}\left(\frac{\partial x^{\mu}}{\partial x^{v}}+x^{\tau} \Gamma_{\tau v}^{\mu}\right)+\left(\frac{\partial x^{l}}{\partial x^{v}}+x^{\tau} \Gamma_{\tau v}^{l}\right) \Gamma_{t \sigma}^{\mu}-\left(\frac{\partial x^{\mu}}{\partial x^{l}}+x^{\tau} \Gamma_{\tau l}^{\mu}\right) \Gamma_{v \sigma}^{\imath} \\
& =\frac{\partial^{2} x^{\mu}}{\partial x^{v} \partial x^{\sigma}}+\frac{\partial}{\partial x^{\sigma}}\left(x^{\tau} \frac{1}{2} g^{\epsilon \mu}\left(\frac{\partial g_{\tau \epsilon}}{\partial x^{v}}+\frac{\partial g_{v \epsilon}}{\partial x^{\tau}}-\frac{\partial g_{\tau v}}{\partial x^{\epsilon}}\right)\right) \\
& +\frac{\partial x^{l}}{\partial x^{v}} \frac{1}{2} g^{\epsilon \mu}\left(\frac{\partial g_{l \epsilon}}{\partial x^{\sigma}}+\frac{\partial g_{\sigma \epsilon}}{\partial x^{l}}-\frac{\partial g_{l \sigma}}{\partial x^{\epsilon}}\right) \\
& +x^{\tau} \frac{1}{2} g^{\epsilon \iota}\left(\frac{\partial g_{\tau \epsilon}}{\partial x^{v}}+\frac{\partial g_{v \epsilon}}{\partial x^{\tau}}-\frac{\partial g_{\tau \nu}}{\partial x^{\epsilon}}\right) \frac{1}{2} g^{\epsilon \mu}\left(\frac{\partial g_{\iota \epsilon}}{\partial x^{\sigma}}+\frac{\partial g_{\sigma \epsilon}}{\partial x^{l}}-\frac{\partial g_{\iota \sigma}}{\partial x^{\epsilon}}\right) \\
& -\frac{\partial x^{\mu}}{\partial x^{l}} \frac{1}{2} g^{\epsilon l}\left(\frac{\partial g_{v \epsilon}}{\partial x^{\sigma}}+\frac{\partial g_{\sigma \epsilon}}{\partial x^{v}}-\frac{\partial g_{v \sigma}}{\partial x^{\epsilon}}\right) \\
& -x^{\tau} \frac{1}{2} g^{\epsilon \mu}\left(\frac{\partial g_{\tau \epsilon}}{\partial x^{l}}+\frac{\partial g_{\iota \epsilon}}{\partial x^{\tau}}-\frac{\partial g_{\tau \iota}}{\partial x^{\epsilon}}\right) \frac{1}{2} g^{\epsilon \iota}\left(\frac{\partial g_{v \epsilon}}{\partial x^{\sigma}}+\frac{\partial g_{\sigma \epsilon}}{\partial x^{\nu}}-\frac{\partial g_{v \sigma}}{\partial x^{\epsilon}}\right) \text {. }
\end{aligned}
$$

Definition 13 The third-order covariant derivative of the contravariant vector satisfied

$$
\begin{aligned}
& x_{; v ; \sigma ; \lambda}^{\mu}=\frac{\partial x_{;, ; \sigma}^{\mu}}{\partial x^{\lambda}}+x_{; v ; \sigma}^{\kappa} \Gamma_{\kappa \lambda}^{\mu}-x_{; \kappa ; \sigma}^{\mu} \Gamma_{v \lambda}^{\kappa}-x_{; v ; \kappa}^{\mu} \Gamma_{\sigma \lambda}^{\kappa} \\
& =\frac{\partial}{\partial x^{\lambda}}\left\{\frac{\partial}{\partial x^{\sigma}}\left(\frac{\partial x^{\mu}}{\partial x^{v}}+x^{\tau} \Gamma_{\tau v}^{\mu}\right)+\left(\frac{\partial x^{l}}{\partial x^{v}}+x^{\tau} \Gamma_{\tau v}^{l}\right) \Gamma_{t \sigma}^{\mu}-\left(\frac{\partial x^{\mu}}{\partial x^{l}}+x^{\tau} \Gamma_{\tau \imath}^{\mu}\right) \Gamma_{v \sigma}^{l}\right\}
\end{aligned}
$$




$$
\begin{aligned}
& +\left\{\frac{\partial}{\partial x^{\sigma}}\left(\frac{\partial x^{\kappa}}{\partial x^{v}}+x^{\tau} \Gamma_{\tau v}^{\kappa}\right)+\left(\frac{\partial x^{l}}{\partial x^{v}}+x^{\tau} \Gamma_{\tau v}^{l}\right) \Gamma_{t \sigma}^{\kappa}-\left(\frac{\partial x^{\kappa}}{\partial x^{l}}+x^{\tau} \Gamma_{\tau l}^{\kappa}\right) \Gamma_{v \sigma}^{l}\right\} \Gamma_{\kappa \lambda}^{\mu} \\
& -\left\{\frac{\partial}{\partial x^{\sigma}}\left(\frac{\partial x^{\mu}}{\partial x^{\kappa}}+x^{\tau} \Gamma_{\tau \kappa}^{\mu}\right)+\left(\frac{\partial x^{l}}{\partial x^{\kappa}}+x^{\tau} \Gamma_{\tau \kappa}^{l}\right) \Gamma_{\imath \sigma}^{\mu}-\left(\frac{\partial x^{\mu}}{\partial x^{l}}+x^{\tau} \Gamma_{\tau l}^{\mu}\right) \Gamma_{\kappa \sigma}^{l}\right\} \Gamma_{\nu \lambda}^{\kappa} \\
& -\left\{\frac{\partial}{\partial x^{\kappa}}\left(\frac{\partial x^{\mu}}{\partial x^{v}}+x^{\tau} \Gamma_{\tau v}^{\mu}\right)+\left(\frac{\partial x^{l}}{\partial x^{v}}+x^{\tau} \Gamma_{\tau v}^{l}\right) \Gamma_{\imath \kappa}^{\mu}-\left(\frac{\partial x^{\mu}}{\partial x^{l}}+x^{\tau} \Gamma_{\tau l}^{\mu}\right) \Gamma_{v \kappa}^{l}\right\} \Gamma_{\sigma \lambda}^{\kappa} \\
& =\frac{\partial^{3} x^{\mu}}{\partial x^{v} \partial x^{\sigma} \partial x^{\lambda}}+\frac{\partial^{2}}{\partial x^{\sigma} \partial x^{\lambda}}\left(x^{\tau} \frac{1}{2} g^{\epsilon \mu}\left(\frac{\partial g_{\tau \epsilon}}{\partial x^{v}}+\frac{\partial g_{v \epsilon}}{\partial x^{\tau}}-\frac{\partial g_{\tau \nu}}{\partial x^{\epsilon}}\right)\right) \\
& +\frac{\partial}{\partial x^{\lambda}}\left(\frac{\partial x^{l}}{\partial x^{v}} \frac{1}{2} g^{\epsilon \mu}\left(\frac{\partial g_{l \epsilon}}{\partial x^{\sigma}}+\frac{\partial g_{\sigma \epsilon}}{\partial x^{l}}-\frac{\partial g_{l \sigma}}{\partial x^{\epsilon}}\right)\right) \\
& +\frac{\partial}{\partial x^{\lambda}}\left(x^{\tau} \frac{1}{2} g^{\epsilon l}\left(\frac{\partial g_{\tau \epsilon}}{\partial x^{v}}+\frac{\partial g_{v \epsilon}}{\partial x^{\tau}}-\frac{\partial g_{\tau \nu}}{\partial x^{\epsilon}}\right) \frac{1}{2} g^{\epsilon \mu}\left(\frac{\partial g_{\iota \epsilon}}{\partial x^{\sigma}}+\frac{\partial g_{\sigma \epsilon}}{\partial x^{l}}-\frac{\partial g_{l \sigma}}{\partial x^{\epsilon}}\right)\right) \\
& -\frac{\partial}{\partial x^{\lambda}}\left(\frac{\partial x^{\mu}}{\partial x^{l}} \frac{1}{2} g^{\epsilon \iota}\left(\frac{\partial g_{v \epsilon}}{\partial x^{\sigma}}+\frac{\partial g_{\sigma \epsilon}}{\partial x^{v}}-\frac{\partial g_{v \sigma}}{\partial x^{\epsilon}}\right)\right) \\
& -\frac{\partial}{\partial x^{\lambda}}\left(x^{\tau} \frac{1}{2} g^{\epsilon \mu}\left(\frac{\partial g_{\tau \epsilon}}{\partial x^{l}}+\frac{\partial g_{\epsilon \epsilon}}{\partial x^{\tau}}-\frac{\partial g_{\tau l}}{\partial x^{\epsilon}}\right) \frac{1}{2} g^{\epsilon \iota}\left(\frac{\partial g_{v \epsilon}}{\partial x^{\sigma}}+\frac{\partial g_{\sigma \epsilon}}{\partial x^{v}}-\frac{\partial g_{v \sigma}}{\partial x^{\epsilon}}\right)\right) \\
& +\frac{\partial^{2} x^{\kappa}}{\partial x^{v} \partial x^{\sigma}} \frac{1}{2} g^{\epsilon \mu}\left(\frac{\partial g_{\kappa \epsilon}}{\partial x^{\lambda}}+\frac{\partial g_{\lambda \epsilon}}{\partial x^{\kappa}}-\frac{\partial g_{\kappa \lambda}}{\partial x^{\epsilon}}\right) \\
& +\frac{\partial}{\partial x^{\sigma}}\left(x^{\tau} \frac{1}{2} g^{\epsilon \kappa}\left(\frac{\partial g_{\tau \epsilon}}{\partial x^{v}}+\frac{\partial g_{v \epsilon}}{\partial x^{\tau}}-\frac{\partial g_{\tau \nu}}{\partial x^{\epsilon}}\right)\right) \frac{1}{2} g^{\epsilon \mu}\left(\frac{\partial g_{\kappa \epsilon}}{\partial x^{\lambda}}+\frac{\partial g_{\lambda \epsilon}}{\partial x^{\kappa}}-\frac{\partial g_{\kappa \lambda}}{\partial x^{\epsilon}}\right) \\
& +\frac{\partial x^{l}}{\partial x^{v}} \frac{1}{2} g^{\epsilon \kappa}\left(\frac{\partial g_{l \epsilon}}{\partial x^{\sigma}}+\frac{\partial g_{\sigma \epsilon}}{\partial x^{l}}-\frac{\partial g_{l \sigma}}{\partial x^{\epsilon}}\right) \frac{1}{2} g^{\epsilon \mu}\left(\frac{\partial g_{\kappa \epsilon}}{\partial x^{\lambda}}+\frac{\partial g_{\lambda \epsilon}}{\partial x^{\kappa}}-\frac{\partial g_{\kappa \lambda}}{\partial x^{\epsilon}}\right) \\
& +x^{\tau} \frac{1}{2} g^{\epsilon l}\left(\frac{\partial g_{\tau \epsilon}}{\partial x^{v}}+\frac{\partial g_{v \epsilon}}{\partial x^{\tau}}-\frac{\partial g_{\tau v}}{\partial x^{\epsilon}}\right) \frac{1}{2} g^{\epsilon \kappa}\left(\frac{\partial g_{l \epsilon}}{\partial x^{\sigma}}+\frac{\partial g_{\sigma \epsilon}}{\partial x^{l}}-\frac{\partial g_{l \sigma}}{\partial x^{\epsilon}}\right) \\
& \times \frac{1}{2} g^{\epsilon \mu}\left(\frac{\partial g_{\kappa \epsilon}}{\partial x^{\lambda}}+\frac{\partial g_{\lambda \epsilon}}{\partial x^{\kappa}}-\frac{\partial g_{\kappa \lambda}}{\partial x^{\epsilon}}\right) \\
& -\frac{\partial x^{\kappa}}{\partial x^{l}} \frac{1}{2} g^{\epsilon l}\left(\frac{\partial g_{v \epsilon}}{\partial x^{\sigma}}+\frac{\partial g_{\sigma \epsilon}}{\partial x^{v}}-\frac{\partial g_{v \sigma}}{\partial x^{\epsilon}}\right) \frac{1}{2} g^{\epsilon \mu}\left(\frac{\partial g_{\kappa \epsilon}}{\partial x^{\lambda}}+\frac{\partial g_{\lambda \epsilon}}{\partial x^{\kappa}}-\frac{\partial g_{\kappa \lambda}}{\partial x^{\epsilon}}\right) \\
& -x^{\tau} \frac{1}{2} g^{\epsilon \kappa}\left(\frac{\partial g_{\tau \epsilon}}{\partial x^{l}}+\frac{\partial g_{\iota \epsilon}}{\partial x^{\tau}}-\frac{\partial g_{\tau \iota}}{\partial x^{\epsilon}}\right) \frac{1}{2} g^{\epsilon \iota}\left(\frac{\partial g_{v \epsilon}}{\partial x^{\sigma}}+\frac{\partial g_{\sigma \epsilon}}{\partial x^{v}}-\frac{\partial g_{v \sigma}}{\partial x^{\epsilon}}\right) \\
& \times \frac{1}{2} g^{\epsilon \mu}\left(\frac{\partial g_{\kappa \epsilon}}{\partial x^{\lambda}}+\frac{\partial g_{\lambda \epsilon}}{\partial x^{\kappa}}-\frac{\partial g_{\kappa \lambda}}{\partial x^{\epsilon}}\right)-\frac{\partial^{2} x^{\mu}}{\partial x^{\kappa} \partial x^{\sigma}} \frac{1}{2} g^{\epsilon \kappa}\left(\frac{\partial g_{v \epsilon}}{\partial x^{\lambda}}+\frac{\partial g_{\lambda \epsilon}}{\partial x^{\nu}}-\frac{\partial g_{\nu \lambda}}{\partial x^{\epsilon}}\right) \\
& -\frac{\partial}{\partial x^{\sigma}}\left(x^{\tau} \frac{1}{2} g^{\epsilon \mu}\left(\frac{\partial g_{\tau \epsilon}}{\partial x^{\kappa}}+\frac{\partial g_{\kappa \epsilon}}{\partial x^{\tau}}-\frac{\partial g_{\tau \kappa}}{\partial x^{\epsilon}}\right)\right) \frac{1}{2} g^{\epsilon \kappa}\left(\frac{\partial g_{v \epsilon}}{\partial x^{\lambda}}+\frac{\partial g_{\lambda \epsilon}}{\partial x^{v}}-\frac{\partial g_{v \lambda}}{\partial x^{\epsilon}}\right) \\
& -\frac{\partial x^{l}}{\partial x^{\kappa}} \frac{1}{2} g^{\epsilon \mu}\left(\frac{\partial g_{l \epsilon}}{\partial x^{\sigma}}+\frac{\partial g_{\sigma \epsilon}}{\partial x^{l}}-\frac{\partial g_{l \sigma}}{\partial x^{\epsilon}}\right) \frac{1}{2} g^{\epsilon \kappa}\left(\frac{\partial g_{v \epsilon}}{\partial x^{\lambda}}+\frac{\partial g_{\lambda \epsilon}}{\partial x^{v}}-\frac{\partial g_{v \lambda}}{\partial x^{\epsilon}}\right) \\
& -x^{\tau} \frac{1}{2} g^{\epsilon \iota}\left(\frac{\partial g_{\tau \epsilon}}{\partial x^{\kappa}}+\frac{\partial g_{\kappa \epsilon}}{\partial x^{\tau}}-\frac{\partial g_{\tau \kappa}}{\partial x^{\epsilon}}\right) \frac{1}{2} g^{\epsilon \mu}\left(\frac{\partial g_{l \epsilon}}{\partial x^{\sigma}}+\frac{\partial g_{\sigma \epsilon}}{\partial x^{l}}-\frac{\partial g_{l \sigma}}{\partial x^{\epsilon}}\right) \\
& \times \frac{1}{2} g^{\epsilon \kappa}\left(\frac{\partial g_{v \epsilon}}{\partial x^{\lambda}}+\frac{\partial g_{\lambda \epsilon}}{\partial x^{v}}-\frac{\partial g_{v \lambda}}{\partial x^{\epsilon}}\right)
\end{aligned}
$$




$$
\begin{aligned}
& +\frac{\partial x^{\mu}}{\partial x^{l}} \frac{1}{2} g^{\epsilon \iota}\left(\frac{\partial g_{\kappa \epsilon}}{\partial x^{\sigma}}+\frac{\partial g_{\sigma \epsilon}}{\partial x^{\kappa}}-\frac{\partial g_{\kappa \sigma}}{\partial x^{\epsilon}}\right) \frac{1}{2} g^{\epsilon \kappa}\left(\frac{\partial g_{v \epsilon}}{\partial x^{\lambda}}+\frac{\partial g_{\lambda \epsilon}}{\partial x^{\nu}}-\frac{\partial g_{v \lambda}}{\partial x^{\epsilon}}\right) \\
& +x^{\tau} \frac{1}{2} g^{\epsilon \mu}\left(\frac{\partial g_{\tau \epsilon}}{\partial x^{l}}+\frac{\partial g_{\iota \epsilon}}{\partial x^{\tau}}-\frac{\partial g_{\tau l}}{\partial x^{\epsilon}}\right) \frac{1}{2} g^{\epsilon \iota}\left(\frac{\partial g_{\kappa \epsilon}}{\partial x^{\sigma}}+\frac{\partial g_{\sigma \epsilon}}{\partial x^{\kappa}}-\frac{\partial g_{\kappa \sigma}}{\partial x^{\epsilon}}\right) \\
& \times \frac{1}{2} g^{\epsilon \kappa}\left(\frac{\partial g_{v \epsilon}}{\partial x^{\lambda}}+\frac{\partial g_{\lambda \epsilon}}{\partial x^{v}}-\frac{\partial g_{v \lambda}}{\partial x^{\epsilon}}\right)-\frac{\partial^{2} x^{\mu}}{\partial x^{v} \partial x^{\kappa}} \frac{1}{2} g^{\epsilon \kappa}\left(\frac{\partial g_{\sigma \epsilon}}{\partial x^{\lambda}}+\frac{\partial g_{\lambda \epsilon}}{\partial x^{\sigma}}-\frac{\partial g_{\sigma \lambda}}{\partial x^{\epsilon}}\right) \\
& -\frac{\partial}{\partial x^{\kappa}}\left(x^{\tau} \frac{1}{2} g^{\epsilon \mu}\left(\frac{\partial g_{\tau \epsilon}}{\partial x^{v}}+\frac{\partial g_{v \epsilon}}{\partial x^{\tau}}-\frac{\partial g_{\tau \nu}}{\partial x^{\epsilon}}\right)\right) \frac{1}{2} g^{\epsilon \kappa}\left(\frac{\partial g_{\sigma \epsilon}}{\partial x^{\lambda}}+\frac{\partial g_{\lambda \epsilon}}{\partial x^{\sigma}}-\frac{\partial g_{\sigma \lambda}}{\partial x^{\epsilon}}\right) \\
& -\frac{\partial x^{l}}{\partial x^{v}} \frac{1}{2} g^{\epsilon \mu}\left(\frac{\partial g_{\iota \epsilon}}{\partial x^{\kappa}}+\frac{\partial g_{\kappa \epsilon}}{\partial x^{l}}-\frac{\partial g_{\iota \kappa}}{\partial x^{\epsilon}}\right) \frac{1}{2} g^{\epsilon \kappa}\left(\frac{\partial g_{\sigma \epsilon}}{\partial x^{\lambda}}+\frac{\partial g_{\lambda \epsilon}}{\partial x^{\sigma}}-\frac{\partial g_{\sigma \lambda}}{\partial x^{\epsilon}}\right) \\
& -x^{\tau} \frac{1}{2} g^{\epsilon l}\left(\frac{\partial g_{\tau \epsilon}}{\partial x^{v}}+\frac{\partial g_{v \epsilon}}{\partial x^{\tau}}-\frac{\partial g_{\tau \nu}}{\partial x^{\epsilon}}\right) \frac{1}{2} g^{\epsilon \mu}\left(\frac{\partial g_{\iota \epsilon}}{\partial x^{\kappa}}+\frac{\partial g_{\kappa \epsilon}}{\partial x^{l}}-\frac{\partial g_{l \kappa}}{\partial x^{\epsilon}}\right) \\
& \times \frac{1}{2} g^{\epsilon \kappa}\left(\frac{\partial g_{\sigma \epsilon}}{\partial x^{\lambda}}+\frac{\partial g_{\lambda \epsilon}}{\partial x^{\sigma}}-\frac{\partial g_{\sigma \lambda}}{\partial x^{\epsilon}}\right) \\
& +\frac{\partial x^{\mu}}{\partial x^{l}} \frac{1}{2} g^{\epsilon l}\left(\frac{\partial g_{v \epsilon}}{\partial x^{\kappa}}+\frac{\partial g_{\kappa \epsilon}}{\partial x^{v}}-\frac{\partial g_{v \kappa}}{\partial x^{\epsilon}}\right) \frac{1}{2} g^{\epsilon \kappa}\left(\frac{\partial g_{\sigma \epsilon}}{\partial x^{\lambda}}+\frac{\partial g_{\lambda \epsilon}}{\partial x^{\sigma}}-\frac{\partial g_{\sigma \lambda}}{\partial x^{\epsilon}}\right) \\
& +x^{\tau} \frac{1}{2} g^{\epsilon \mu}\left(\frac{\partial g_{\tau \epsilon}}{\partial x^{l}}+\frac{\partial g_{\iota \epsilon}}{\partial x^{\tau}}-\frac{\partial g_{\tau \iota}}{\partial x^{\epsilon}}\right) \frac{1}{2} g^{\epsilon \iota}\left(\frac{\partial g_{v \epsilon}}{\partial x^{\kappa}}+\frac{\partial g_{\kappa \epsilon}}{\partial x^{v}}-\frac{\partial g_{v \kappa}}{\partial x^{\epsilon}}\right) \\
& \times \frac{1}{2} g^{\epsilon \kappa}\left(\frac{\partial g_{\sigma \epsilon}}{\partial x^{\lambda}}+\frac{\partial g_{\lambda \epsilon}}{\partial x^{\sigma}}-\frac{\partial g_{\sigma \lambda}}{\partial x^{\epsilon}}\right) \text {. }
\end{aligned}
$$

Definition 14 When the next conversion equation is established, $x_{\mu}^{\mu}$ is components of a tensor of rank zero. $x_{\mu}^{\mu}=\frac{\partial x^{\mu}}{\partial x^{v}} \frac{\partial x^{v}}{\partial x^{\mu}} x_{v}^{v}$

Definition 15 When the next conversion equation is established, $x^{\mu}$ is contravariant components of a tensor of the first rank [4]. $x^{\mu}=\frac{\partial x^{\mu}}{\partial x^{v}} x^{v}$

Definition 16 When the next conversion equation is established, $x_{\mu}$ is covariant components of a tensor of the first rank [4]. $x_{\mu}=\frac{\partial x^{v}}{\partial x^{\mu}} x_{v}$

Definition 17 When the next conversion equation is established, $x^{\mu v}$ is contravariant components of a tensor of the second rank [4]. $x^{\mu \nu}=\frac{\partial x^{\mu}}{\partial x^{\sigma}} \frac{\partial x^{\nu}}{\partial x^{\lambda}} x^{\sigma \lambda}$

Definition 18 When the next conversion equation is established, $x_{\mu v}$ is covariant components of a tensor of the second rank [4]. $x_{\mu \nu}=\frac{\partial x^{\sigma}}{\partial x^{\mu}} \frac{\partial x^{\lambda}}{\partial x^{\nu}} x_{\sigma \lambda}$

Definition 19 When the next conversion equation is established, $x_{v}^{\mu}$ is components of the mixed tensor of the second rank [4]. $x_{v}^{\mu}=\frac{\partial x^{\mu}}{\partial x^{\sigma}} \frac{\partial x^{\lambda}}{\partial x^{v}} x_{\lambda}^{\sigma}$

Definition 20 When the next conversion equation is established, $x_{v \sigma}^{\mu}$ is components of the mixed tensor of the third rank of the second rank covariant in the first rank contravariant [4]. $x_{v \sigma}^{\mu}=\frac{\partial x^{\mu}}{\partial x^{\lambda}} \frac{\partial x^{l}}{\partial x^{v}} \frac{\partial x^{\epsilon}}{\partial x^{\sigma}} x_{t \epsilon}^{\lambda}$

Definition 21 When the next conversion equation is established, $x_{v \sigma \lambda}^{\mu}$ is 
components of the mixed tensor of the fourth rank of the third rank covariant in the first rank contravariant. $x_{v \sigma \lambda}^{\mu}=\frac{\partial x^{\mu}}{\partial x^{l}} \frac{\partial x^{\epsilon}}{\partial x^{\nu}} \frac{\partial x^{\alpha}}{\partial x^{\sigma}} \frac{\partial x^{\beta}}{\partial x^{\lambda}} x_{\epsilon \alpha \beta}^{l}$

\section{About Covariant Derivative for the Scalar in Tensor Satisfying Binary Law}

Proposition 1 When all coordinate systems satisfy Binary Law,

$M_{; v}=\frac{\partial M}{\partial x^{v}}=0$ is established for $\frac{\partial^{3} x^{\mu}}{\partial x^{v} \partial x^{v} \partial x^{v}}=M$.

Proof: I get

$$
M_{; v}=\frac{\partial M}{\partial x^{v}}=0
$$

as $\epsilon=1$ for Definition 9, Hypothesis 1 .

-End Proof-

Proposition 2 When all coordinate systems satisfy Binary Law, $\frac{\partial^{2} x_{\mu}}{\partial x^{v} \partial x^{v}}=0$ is established.

Proof: I get

$$
\frac{\partial^{2} x_{\mu}}{\partial x^{v} \partial x^{v}}=0
$$

from (1), (77).

-End Proof-

Proposition 3 When all coordinate systems satisfy Binary Law, $\frac{\partial^{4} x^{\mu}}{\partial x^{v} \partial x^{v} \partial x^{v} \partial x^{v}}=0$ is established.

Proof: I get

$$
\frac{\partial^{4} x^{\mu}}{\partial x^{v} \partial x^{v} \partial x^{v} \partial x^{v}}=0
$$

from (1), Definition 8.

-End Proof-

\section{About Covariant Derivative for the Vector in Tensor Satisfying Binary Law}

Proposition $4 x_{\mu ; \nu}=\frac{\partial x_{\mu}}{\partial x^{v}}, x_{\mu}^{; \mu}=\frac{\partial x_{\mu}}{\partial x_{\mu}}-x_{v} \frac{1}{2}\left(\frac{\partial g^{v \mu}}{\partial x^{\mu}}\right)$ is established in tensor satisfying Binary Law.

Proof: If all coordinate systems satisfy Binary Law, I get

$$
\begin{aligned}
x_{\mu ; \nu} & =\frac{\partial x_{\mu}}{\partial x^{v}}-x_{v} \frac{1}{2} g^{\nu v}\left(\frac{\partial g_{\mu \nu}}{\partial x^{v}}+\frac{\partial g_{v v}}{\partial x^{\mu}}-\frac{\partial g_{\mu \nu}}{\partial x^{v}}\right) \\
& =\frac{\partial x_{\mu}}{\partial x^{v}}-x_{v} \frac{1}{2}\left(\frac{\partial g_{v}^{v}}{\partial x^{\mu}}\right)
\end{aligned}
$$

from Definition 10. (4) must rewrite it in 


$$
x_{\mu ; \nu}=\frac{\partial x_{\mu}}{\partial x^{v}}-x_{\sigma} \frac{1}{2}\left(\frac{\partial g_{v}^{\sigma}}{\partial x^{\mu}}\right)
$$

by (4) being a tensor equation. The dummy index has an invariable property for consideration of Binary Law. In other words, the index which was dummy index in Definition 10 is dummy index in (5). I get the conclusion that (5) doesn't satisfy Binary Law from Definition 6. I get the conclusion that Definition 10 isn't an equation of the tensor satisfying Binary Law because (5) doesn't satisfy Binary Law.

I rewrite one existing index $v$ in each term of (5) in index $\mu$ using Definition 2 and get

$$
\begin{aligned}
& x_{\mu}^{; \mu}=\frac{\partial x_{\mu}}{\partial x_{\mu}}-x_{\sigma} \frac{1}{2}\left(\frac{\partial g^{\sigma \mu}}{\partial x^{\mu}}\right), \\
& x_{\mu}^{; \mu}=\frac{\partial x_{\mu}}{\partial x_{\mu}}-x_{v} \frac{1}{2}\left(\frac{\partial g^{v \mu}}{\partial x^{\mu}}\right) .
\end{aligned}
$$

I rewrite one existing index $v$ in each term of (5) in index $\mu$ using Definition 4 and get

$$
-x_{\mu ; \mu}=-\frac{\partial x_{\mu}}{\partial x^{\mu}}+x_{\sigma} \frac{1}{2}\left(\frac{\partial g_{\mu}^{\sigma}}{\partial x^{\mu}}\right)
$$

I get

$$
-x_{\mu ; \mu}=-\frac{\partial x_{\mu}}{\partial x^{\mu}}
$$

in consideration of Definition 7 for (7). Because the second term of the right side of (8) doesn't exist,

$$
x_{\mu ; v}=\frac{\partial x_{\mu}}{\partial x^{v}}
$$

can rewrite (8) using Definition 4. In addition, $x_{\mu ; \nu}$ can't rewrite $x_{\mu}^{; \mu}$ of (6) using Definition 2 because the second term of the right side exists in (6).

-End Proof-

Proposition $5 x_{; v}^{\mu}=\frac{\partial x^{\mu}}{\partial x^{v}}$ is established in tensor satisfying Binary Law.

Proof: If all coordinate systems satisfy Binary Law, I get

$$
\begin{aligned}
x_{; v}^{\mu} & =\frac{\partial x^{\mu}}{\partial x^{v}}+x^{v} \frac{1}{2} g^{v \mu}\left(\frac{\partial \widehat{g_{v v}}}{\partial x^{v}}+\frac{\partial g_{v v}}{\partial x^{v}}-\frac{\partial g_{v v}}{\partial x^{v}}\right) \\
& =\frac{\partial x^{\mu}}{\partial x^{v}}+x^{v} \frac{1}{2}\left(\frac{\partial \widehat{g_{v}^{\mu}}}{\partial x^{v}}\right) \\
& =\frac{\partial x^{\mu}}{\partial x^{v}}+x^{v} \frac{1}{2}\left(\frac{\partial g_{v}^{\mu}}{\partial x^{v}}\right)
\end{aligned}
$$

from Definition 11. (10), (11) must rewrite it in 


$$
\begin{aligned}
x_{; v}^{\mu} & =\frac{\partial x^{\mu}}{\partial x^{\nu}}+x^{\sigma} \frac{1}{2}\left(\frac{\partial g_{\sigma}^{\mu}}{\partial x^{v}}\right) \\
& =\frac{\partial x^{\mu}}{\partial x^{\nu}}+x^{\sigma} \frac{1}{2}\left(\frac{\partial g_{v}^{\mu}}{\partial x^{\sigma}}\right)
\end{aligned}
$$

by (10), (11) being a tensor equation.

The dummy index has an invariable property for consideration of Binary Law. In other words, the index which was dummy index in Definition 11 is dummy index in (12), (13). I get the conclusion that (12), (13) doesn't satisfy Binary Law from Definition 6. I get the conclusion that Definition 11 isn't an equation of the tensor satisfying Binary Law because (12), (13) doesn't satisfy Binary Law.

I rewrite one existing index $v$ in each term of (12), (13) in index $\mu$ using Definition 4 and get

$$
\begin{aligned}
-x_{; \mu}^{\mu} & =-\frac{\partial x^{\mu}}{\partial x^{\mu}}-x^{\sigma} \frac{1}{2}\left(\frac{\partial g_{\sigma}^{\mu}}{\partial x^{\mu}}\right) \\
& =-\frac{\partial x^{\mu}}{\partial x^{\mu}}-x^{\sigma} \frac{1}{2}\left(\frac{\partial g_{\mu}^{\mu}}{\partial x^{\sigma}}\right) .
\end{aligned}
$$

I get

$$
-x_{; \mu}^{\mu}=-\frac{\partial x^{\mu}}{\partial x^{\mu}}
$$

in consideration of Definition 7 for (14). Because the second term of the right side of (15) doesn't exist,

$$
x_{; v}^{\mu}=\frac{\partial x^{\mu}}{\partial x^{v}}
$$

can rewrite (15) using Definition 4. I rewrite one existing index $v$ in each term of (12), (13) in index $\mu$ using Definition 2 and get

$$
\begin{aligned}
x^{\mu ; \mu} & =\frac{\partial x^{\mu}}{\partial x_{\mu}}+x^{\sigma} \frac{1}{2}\left(\frac{\partial g_{\sigma}^{\mu}}{\partial x_{\mu}}\right) \\
& =\frac{\partial x^{\mu}}{\partial x_{\mu}}+x^{\sigma} \frac{1}{2}\left(\frac{\partial g^{\mu \mu}}{\partial x^{\sigma}}\right) .
\end{aligned}
$$

-End Proof-

Proposition $6 x_{; v: v}^{\mu}=\frac{\partial^{2} x^{\mu}}{\partial x^{v} \partial x^{v}}$ is established in tensor satisfying Binary Law.

Proof: If all coordinate systems satisfy Binary Law, I get

$$
\begin{aligned}
x_{; v ; v}^{\mu}= & \frac{\partial^{2} x^{\mu}}{\partial x^{v} \partial x^{v}}+\frac{\partial}{\partial x^{v}}\left(x^{v} \frac{1}{2} g^{v \mu}\left(\frac{\partial \widehat{g_{v v}}}{\partial x^{v}}+\frac{\partial g_{v v}}{\partial x^{v}}-\frac{\partial g_{v v}}{\partial x^{v}}\right)\right) \\
& +\frac{\partial x^{v}}{\partial x^{v}} \frac{1}{2} g^{v \mu}\left(\frac{\partial \widehat{g_{v v}}}{\partial x^{v}}+\frac{\partial g_{v v}}{\partial x^{v}}-\frac{\partial g_{v v}}{\partial x^{v}}\right)
\end{aligned}
$$




$$
\begin{aligned}
+ & x^{v} \frac{1}{2} g^{v v}\left(\frac{\partial \widehat{g_{v v}}}{\partial x^{v}}+\frac{\partial g_{v v}}{\partial x^{v}}-\frac{\partial g_{v v}}{\partial x^{v}}\right) \frac{1}{2} g^{v \mu}\left(\frac{\partial \widehat{g_{v v}}}{\partial x^{v}}+\frac{\partial g_{v v}}{\partial x^{v}}-\frac{\partial g_{v v}}{\partial x^{v}}\right) \\
- & \frac{\partial x^{\mu}}{\partial x^{v}} \frac{1}{2} g^{v v}\left(\frac{\partial \widehat{g_{v v}}}{\partial x^{v}}+\frac{\partial g_{v v}}{\partial x^{v}}-\frac{\partial g_{v v}}{\partial x^{v}}\right) \\
& -x^{v} \frac{1}{2} g^{v \mu}\left(\frac{\partial \widehat{g_{v v}}}{\partial x^{v}}+\frac{\partial g_{v v}}{\partial x^{v}}-\frac{\partial g_{v v}}{\partial x^{v}}\right) \frac{1}{2} g^{v v}\left(\frac{\partial \widehat{g_{v v}}}{\partial x^{v}}+\frac{\partial g_{v v}}{\partial x^{v}}-\frac{\partial g_{v v}}{\partial x^{v}}\right) \\
= & \frac{\partial^{2} x^{\mu}}{\partial x^{v} \partial x^{v}}+\frac{\partial}{\partial x^{v}}\left(x^{v} \frac{1}{2}\left(\frac{\partial g_{v}^{\mu}}{\partial x^{v}}\right)\right)+\frac{\partial x^{v}}{\partial x^{v}} \frac{1}{2}\left(\frac{\partial g_{v}^{\mu}}{\partial x^{v}}\right) \\
+ & x^{v} \frac{1}{2}\left(\frac{\partial g_{v}^{v}}{\partial x^{v}}\right) \frac{1}{2}\left(\frac{\partial g_{v}^{\mu}}{\partial x^{v}}\right)-\frac{\partial x^{\mu}}{\partial x^{v}} \frac{1}{2}\left(\frac{\partial g_{v}^{v}}{\partial x^{v}}\right)-x^{v} \frac{1}{2}\left(\frac{\partial g_{v}^{\mu}}{\partial x^{v}}\right) \frac{1}{2}\left(\frac{\partial g_{v}^{v}}{\partial x^{v}}\right) \\
= & \frac{\partial^{2} x^{\mu}}{\partial x^{v} \partial x^{v}}+\frac{\partial}{\partial x^{v}}\left(x^{v} \frac{1}{2}\left(\frac{\partial g_{v}^{\mu}}{\partial x^{v}}\right)\right)+\frac{\partial x^{v}}{\partial x^{v}} \frac{1}{2}\left(\frac{\partial g_{v}^{\mu}}{\partial x^{v}}\right) \\
& +x^{v} \frac{1}{2}\left(\frac{\partial g_{v}^{v}}{\partial x^{v}}\right) \frac{1}{2}\left(\frac{\partial g_{v}^{\mu}}{\partial x^{v}}\right)-\frac{\partial x^{\mu}}{\partial x^{v}} \frac{1}{2}\left(\frac{\partial g_{v}^{v}}{\partial x^{v}}\right)-x^{v} \frac{1}{2}\left(\frac{\partial g_{v}^{\mu}}{\partial x^{v}}\right) \frac{1}{2}\left(\frac{\partial g_{v}^{v}}{\partial x^{v}}\right)
\end{aligned}
$$

from Definition 12. (17), (18) must rewrite it in

$$
\begin{aligned}
x_{; v ; v}^{\mu}= & \frac{\partial^{2} x^{\mu}}{\partial x^{v} \partial x^{v}}+\frac{\partial}{\partial x^{v}}\left(x^{\sigma} \frac{1}{2}\left(\frac{\partial g_{\sigma}^{\mu}}{\partial x^{v}}\right)\right)+\frac{\partial x^{\sigma}}{\partial x^{v}} \frac{1}{2}\left(\frac{\partial g_{\sigma}^{\mu}}{\partial x^{v}}\right) \\
& +x^{\sigma} \frac{1}{2}\left(\frac{\partial g_{\sigma}^{\sigma}}{\partial x^{v}}\right) \frac{1}{2}\left(\frac{\partial g_{\sigma}^{\mu}}{\partial x^{v}}\right)-\frac{\partial x^{\mu}}{\partial x^{\sigma}} \frac{1}{2}\left(\frac{\partial g_{v}^{\sigma}}{\partial x^{v}}\right)-x^{\sigma} \frac{1}{2}\left(\frac{\partial g_{\sigma}^{\mu}}{\partial x^{\sigma}}\right) \frac{1}{2}\left(\frac{\partial g_{v}^{\sigma}}{\partial x^{v}}\right) \\
= & \frac{\partial^{2} x^{\mu}}{\partial x^{v} \partial x^{v}}+\frac{\partial}{\partial x^{v}}\left(x^{\sigma} \frac{1}{2}\left(\frac{\partial g_{v}^{\mu}}{\partial x^{\sigma}}\right)\right)+\frac{\partial x^{\sigma}}{\partial x^{v}} \frac{1}{2}\left(\frac{\partial g_{v}^{\mu}}{\partial x^{\sigma}}\right) \\
+ & x^{\sigma} \frac{1}{2}\left(\frac{\partial g_{v}^{\sigma}}{\partial x^{\sigma}}\right) \frac{1}{2}\left(\frac{\partial g_{v}^{\mu}}{\partial x^{\sigma}}\right)-\frac{\partial x^{\mu}}{\partial x^{\sigma}} \frac{1}{2}\left(\frac{\partial g_{v}^{\sigma}}{\partial x^{v}}\right)-x^{\sigma} \frac{1}{2}\left(\frac{\partial g_{\sigma}^{\mu}}{\partial x^{\sigma}}\right) \frac{1}{2}\left(\frac{\partial g_{v}^{\sigma}}{\partial x^{v}}\right)
\end{aligned}
$$

by (17), (18) being a tensor equation. The dummy index has an invariable property for consideration of Binary Law. In other words, the index which was dummy index in Definition 12 is dummy index in (19), (20). I get the conclusion that (19), (20) doesn't satisfy Binary Law from Definition 6. I get the conclusion that Definition 12 isn't an equation of the tensor satisfying Binary Law because (19), (20) doesn't satisfy Binary Law.

I rewrite two existing index $v$ in each term of (19), (20) in index $\mu$ using Definition 4 and get

$$
\begin{aligned}
x_{; \mu ; \mu}^{\mu}= & \frac{\partial^{2} x^{\mu}}{\partial x^{\mu} \partial x^{\mu}}+\frac{\partial}{\partial x^{\mu}}\left(x^{\sigma} \frac{1}{2}\left(\frac{\partial g_{\sigma}^{\mu}}{\partial x^{\mu}}\right)\right)+\frac{\partial x^{\sigma}}{\partial x^{\mu}} \frac{1}{2}\left(\frac{\partial g_{\sigma}^{\mu}}{\partial x^{\mu}}\right) \\
& +x^{\sigma} \frac{1}{2}\left(\frac{\partial g_{\sigma}^{\sigma}}{\partial x^{\mu}}\right) \frac{1}{2}\left(\frac{\partial g_{\sigma}^{\mu}}{\partial x^{\mu}}\right)-\frac{\partial x^{\mu}}{\partial x^{\sigma}} \frac{1}{2}\left(\frac{\partial g_{\mu}^{\sigma}}{\partial x^{\mu}}\right)-x^{\sigma} \frac{1}{2}\left(\frac{\partial g_{\sigma}^{\mu}}{\partial x^{\sigma}}\right) \frac{1}{2}\left(\frac{\partial g_{\mu}^{\sigma}}{\partial x^{\mu}}\right) \\
= & \frac{\partial^{2} x^{\mu}}{\partial x^{\mu} \partial x^{\mu}}+\frac{\partial}{\partial x^{\mu}}\left(x^{\sigma} \frac{1}{2}\left(\frac{\partial g_{\mu}^{\mu}}{\partial x^{\sigma}}\right)\right)+\frac{\partial x^{\sigma}}{\partial x^{\mu}} \frac{1}{2}\left(\frac{\partial g_{\mu}^{\mu}}{\partial x^{\sigma}}\right) \\
& +x^{\sigma} \frac{1}{2}\left(\frac{\partial g_{\mu}^{\sigma}}{\partial x^{\sigma}}\right) \frac{1}{2}\left(\frac{\partial g_{\mu}^{\mu}}{\partial x^{\sigma}}\right)-\frac{\partial x^{\mu}}{\partial x^{\sigma}} \frac{1}{2}\left(\frac{\partial g_{\mu}^{\sigma}}{\partial x^{\mu}}\right)-x^{\sigma} \frac{1}{2}\left(\frac{\partial g_{\sigma}^{\mu}}{\partial x^{\sigma}}\right) \frac{1}{2}\left(\frac{\partial g_{\mu}^{\sigma}}{\partial x^{\mu}}\right) .
\end{aligned}
$$


I get

$$
x_{; \mu ; \mu}^{\mu}=\frac{\partial^{2} x^{\mu}}{\partial x^{\mu} \partial x^{\mu}}
$$

in consideration of Definition 7 for (21). Because the second term of the right side of (22) doesn't exist,

$$
x_{; v: v}^{\mu}=\frac{\partial^{2} x^{\mu}}{\partial x^{v} \partial x^{v}}
$$

can rewrite (22) using Definition 4. I rewrite two existing index $v$ in each term of (19), (20) in index $\mu$ using Definition 2 and get

$$
\begin{aligned}
x^{\mu ; \mu ; \mu}= & \frac{\partial^{2} x^{\mu}}{\partial x_{\mu} \partial x_{\mu}}+\frac{\partial}{\partial x_{\mu}}\left(x^{\sigma} \frac{1}{2}\left(\frac{\partial g_{\sigma}^{\mu}}{\partial x_{\mu}}\right)\right)+\frac{\partial x^{\sigma}}{\partial x_{\mu}} \frac{1}{2}\left(\frac{\partial g_{\sigma}^{\mu}}{\partial x_{\mu}}\right) \\
& +x^{\sigma} \frac{1}{2}\left(\frac{\partial g_{\sigma}^{\sigma}}{\partial x_{\mu}}\right) \frac{1}{2}\left(\frac{\partial g_{\sigma}^{\mu}}{\partial x_{\mu}}\right)-\frac{\partial x^{\mu}}{\partial x^{\sigma}} \frac{1}{2}\left(\frac{\partial g^{\sigma \mu}}{\partial x_{\mu}}\right)-x^{\sigma} \frac{1}{2}\left(\frac{\partial g_{\sigma}^{\mu}}{\partial x^{\sigma}}\right) \frac{1}{2}\left(\frac{\partial g^{\sigma \mu}}{\partial x_{\mu}}\right) \\
= & \frac{\partial^{2} x^{\mu}}{\partial x_{\mu} \partial x_{\mu}}+\frac{\partial}{\partial x_{\mu}}\left(x^{\sigma} \frac{1}{2}\left(\frac{\partial g^{\mu \mu}}{\partial x^{\sigma}}\right)\right)+\frac{\partial x^{\sigma}}{\partial x_{\mu}} \frac{1}{2}\left(\frac{\partial g^{\mu \mu}}{\partial x^{\sigma}}\right) \\
& +x^{\sigma} \frac{1}{2}\left(\frac{\partial g^{\sigma \mu}}{\partial x^{\sigma}}\right) \frac{1}{2}\left(\frac{\partial g^{\mu \mu}}{\partial x^{\sigma}}\right)-\frac{\partial x^{\mu}}{\partial x^{\sigma}} \frac{1}{2}\left(\frac{\partial g^{\sigma \mu}}{\partial x_{\mu}}\right)-x^{\sigma} \frac{1}{2}\left(\frac{\partial g_{\sigma}^{\mu}}{\partial x^{\sigma}}\right) \frac{1}{2}\left(\frac{\partial g^{\sigma \mu}}{\partial x_{\mu}}\right) .
\end{aligned}
$$

-End Proof-

Proposition $7 x_{; ; ; ; v v}^{\mu}=\frac{\partial^{3} x^{\mu}}{\partial x^{v} \partial x^{v} \partial x^{v}}$ is established in tensor satisfying Binary Law.

Proof: If all coordinate systems satisfy Binary Law, I get

$$
\begin{aligned}
& x_{; \nu ;, ; \nu}^{\mu}=\frac{\partial^{3} x^{\mu}}{\partial x^{v} \partial x^{v} \partial x^{v}}+\frac{\partial^{2}}{\partial x^{v} \partial x^{v}}\left(x^{v} \frac{1}{2} g^{v \mu}\left(\frac{\partial \widehat{g_{v v}}}{\partial x^{v}}+\frac{\partial g_{v v}}{\partial x^{v}}-\frac{\partial g_{v v}}{\partial x^{v}}\right)\right) \\
& +\frac{\partial}{\partial x^{v}}\left(\frac{\partial x^{v}}{\partial x^{v}} \frac{1}{2} g^{v \mu}\left(\frac{\partial \widehat{g_{v v}}}{\partial x^{v}}+\frac{\partial g_{v v}}{\partial x^{v}}-\frac{\partial g_{v^{v}}}{\partial x^{v}}\right)\right) \\
& +\frac{\partial}{\partial x^{v}}\left(x^{\nu} \frac{1}{2} g^{v v}\left(\frac{\partial \widehat{g_{v v}}}{\partial x^{v}}+\frac{\partial g_{v v}}{\partial x^{v}}-\frac{\partial g_{v v}}{\partial x^{v}}\right) \frac{1}{2} g^{v \mu}\left(\frac{\partial g_{v v}}{\partial x^{v}}+\frac{\partial g_{v v}}{\partial x^{v}}-\frac{\partial g_{v v}}{\partial x^{v}}\right)\right) \\
& -\frac{\partial}{\partial x^{v}}\left(\frac{\partial x^{\mu}}{\partial x^{v}} \frac{1}{2} g^{v v}\left(\frac{\partial g_{v v}}{\partial x^{v}}+\frac{\partial g_{v v}}{\partial x^{v}}-\frac{\partial g_{v v}}{\partial x^{v}}\right)\right) \\
& -\frac{\partial}{\partial x^{v}}\left(x^{\nu} \frac{1}{2} g^{v \mu}\left(\frac{\partial \widehat{g_{v v}}}{\partial x^{v}}+\frac{\partial g_{v v}}{\partial x^{v}}-\frac{\partial g_{v v}}{\partial x^{v}}\right) \frac{1}{2} g^{v v}\left(\frac{\partial \widehat{g_{v v}}}{\partial x^{v}}+\frac{\partial g_{v v}}{\partial x^{v}}-\frac{\partial g_{v V}}{\partial x^{v}}\right)\right) \\
& +\frac{\partial^{2} x^{v}}{\partial x^{v} \partial x^{v}} \frac{1}{2} g^{v \mu}\left(\frac{\partial \widehat{g_{v v}}}{\partial x^{v}}+\frac{\partial g_{v v}}{\partial x^{v}}-\frac{\partial g_{v v}}{\partial x^{v}}\right)
\end{aligned}
$$

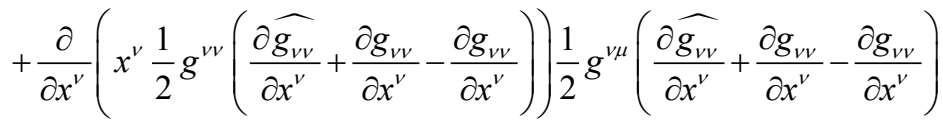




$$
\begin{aligned}
& +\frac{\partial x^{v}}{\partial x^{v}} \frac{1}{2} g^{v v}\left(\frac{\partial \widehat{g_{v v}}}{\partial x^{v}}+\frac{\partial g_{v v}}{\partial x^{v}}-\frac{\partial g_{v v}}{\partial x^{v}}\right) \frac{1}{2} g^{v \mu}\left(\frac{\partial \widehat{g_{v v}}}{\partial x^{v}}+\frac{\partial g_{v v}}{\partial x^{v}}-\frac{\partial g_{v v}}{\partial x^{v}}\right) \\
& +x^{v} \frac{1}{2} g^{v v}\left(\frac{\partial \widehat{g_{v v}}}{\partial x^{v}}+\frac{\partial g_{v v}}{\partial x^{v}}-\frac{\partial g_{v v}}{\partial x^{v}}\right) \frac{1}{2} g^{v v}\left(\frac{\partial \widehat{g_{v v}}}{\partial x^{v}}+\frac{\partial g_{v v}}{\partial x^{v}}-\frac{\partial g_{v v}}{\partial x^{v}}\right) \\
& \times \frac{1}{2} g^{v \mu}\left(\frac{\partial \widehat{g_{v v}}}{\partial x^{v}}+\frac{\partial g_{v v}}{\partial x^{v}}-\frac{\partial g_{v v}}{\partial x^{v}}\right) \\
& -\frac{\partial x^{v}}{\partial x^{v}} \frac{1}{2} g^{v v}\left(\frac{\partial \widehat{g_{v v}}}{\partial x^{v}}+\frac{\partial g_{v v}}{\partial x^{v}}-\frac{\partial g_{v v}}{\partial x^{v}}\right) \frac{1}{2} g^{v \mu}\left(\frac{\partial \widehat{g_{v v}}}{\partial x^{v}}+\frac{\partial g_{v v}}{\partial x^{v}}-\frac{\partial g_{v v}}{\partial x^{v}}\right) \\
& -x^{v} \frac{1}{2} g^{v v}\left(\frac{\partial \widehat{g_{v v}}}{\partial x^{v}}+\frac{\partial g_{v v}}{\partial x^{v}}-\frac{\partial g_{v v}}{\partial x^{v}}\right) \frac{1}{2} g^{v v}\left(\frac{\partial \widehat{g_{v v}}}{\partial x^{v}}+\frac{\partial g_{v v}}{\partial x^{v}}-\frac{\partial g_{v v}}{\partial x^{v}}\right) \\
& \times \frac{1}{2} g^{v \mu}\left(\frac{\partial \widehat{g_{v v}}}{\partial x^{v}}+\frac{\partial g_{v v}}{\partial x^{v}}-\frac{\partial g_{v v}}{\partial x^{v}}\right)-\frac{\partial^{2} x^{\mu}}{\partial x^{v} \partial x^{v}} \frac{1}{2} g^{v v}\left(\frac{\partial \widehat{g_{v v}}}{\partial x^{v}}+\frac{\partial g_{v v}}{\partial x^{v}}-\frac{\partial g_{v v}}{\partial x^{v}}\right) \\
& -\frac{\partial}{\partial x^{v}}\left(x^{\nu} \frac{1}{2} g^{v \mu}\left(\frac{\partial \widehat{g_{v v}}}{\partial x^{v}}+\frac{\partial g_{v v}}{\partial x^{v}}-\frac{\partial g_{v v}}{\partial x^{v}}\right)\right) \frac{1}{2} g^{v v}\left(\frac{\partial \widehat{g_{v v}}}{\partial x^{v}}+\frac{\partial g_{v v}}{\partial x^{v}}-\frac{\partial g_{v v}}{\partial x^{v}}\right) \\
& -\frac{\partial x^{v}}{\partial x^{v}} \frac{1}{2} g^{v \mu}\left(\frac{\partial \widehat{g_{v v}}}{\partial x^{v}}+\frac{\partial g_{v v}}{\partial x^{v}}-\frac{\partial g_{v v}}{\partial x^{v}}\right) \frac{1}{2} g^{v v}\left(\frac{\partial \widehat{g_{v v}}}{\partial x^{v}}+\frac{\partial g_{v v}}{\partial x^{v}}-\frac{\partial g_{v v}}{\partial x^{v}}\right) \\
& -x^{v} \frac{1}{2} g^{v v}\left(\frac{\partial \widehat{g_{v v}}}{\partial x^{v}}+\frac{\partial g_{v v}}{\partial x^{v}}-\frac{\partial g_{v v}}{\partial x^{v}}\right) \frac{1}{2} g^{v \mu}\left(\frac{\partial \widehat{g_{v v}}}{\partial x^{v}}+\frac{\partial g_{v v}}{\partial x^{v}}-\frac{\partial g_{v v}}{\partial x^{v}}\right) \\
& \times \frac{1}{2} g^{v v}\left(\frac{\partial \widehat{g_{v v}}}{\partial x^{v}}+\frac{\partial g_{v v}}{\partial x^{v}}-\frac{\partial g_{v v}}{\partial x^{v}}\right) \\
& +\frac{\partial x^{\mu}}{\partial x^{v}} \frac{1}{2} g^{v v}\left(\frac{\partial \widehat{g_{v v}}}{\partial x^{v}}+\frac{\partial g_{v v}}{\partial x^{v}}-\frac{\partial g_{v v}}{\partial x^{v}}\right) \frac{1}{2} g^{v v}\left(\frac{\partial \widehat{g_{v v}}}{\partial x^{v}}+\frac{\partial g_{v v}}{\partial x^{v}}-\frac{\partial g_{v v}}{\partial x^{v}}\right) \\
& +x^{v} \frac{1}{2} g^{v \mu}\left(\frac{\partial \widehat{g_{v v}}}{\partial x^{v}}+\frac{\partial g_{v v}}{\partial x^{v}}-\frac{\partial g_{v v}}{\partial x^{v}}\right) \frac{1}{2} g^{v v}\left(\frac{\partial \widehat{g_{v v}}}{\partial x^{v}}+\frac{\partial g_{v v}}{\partial x^{v}}-\frac{\partial g_{v v}}{\partial x^{v}}\right) \\
& \times \frac{1}{2} g^{v v}\left(\frac{\partial \widehat{g_{v v}}}{\partial x^{v}}+\frac{\partial g_{v v}}{\partial x^{v}}-\frac{\partial g_{v v}}{\partial x^{v}}\right)-\frac{\partial^{2} x^{\mu}}{\partial x^{v} \partial x^{v}} \frac{1}{2} g^{v v}\left(\frac{\partial \widehat{g_{v v}}}{\partial x^{v}}+\frac{\partial g_{v v}}{\partial x^{v}}-\frac{\partial g_{v v}}{\partial x^{v}}\right) \\
& -\frac{\partial}{\partial x^{v}}\left(x^{v} \frac{1}{2} g^{v \mu}\left(\frac{\partial \widehat{g_{v v}}}{\partial x^{v}}+\frac{\partial g_{v v}}{\partial x^{v}}-\frac{\partial g_{v v}}{\partial x^{v}}\right)\right) \frac{1}{2} g^{v v}\left(\frac{\partial \widehat{g_{v v}}}{\partial x^{v}}+\frac{\partial g_{v v}}{\partial x^{v}}-\frac{\partial g_{v v}}{\partial x^{v}}\right) \\
& -\frac{\partial x^{v}}{\partial x^{v}} \frac{1}{2} g^{v \mu}\left(\frac{\partial \widehat{g_{v v}}}{\partial x^{v}}+\frac{\partial g_{v v}}{\partial x^{v}}-\frac{\partial g_{v v}}{\partial x^{v}}\right) \frac{1}{2} g^{v v}\left(\frac{\partial \widehat{g_{v v}}}{\partial x^{v}}+\frac{\partial g_{v v}}{\partial x^{v}}-\frac{\partial g_{v v}}{\partial x^{v}}\right) \\
& -x^{v} \frac{1}{2} g^{v v}\left(\frac{\partial \widehat{g_{v v}}}{\partial x^{v}}+\frac{\partial g_{v v}}{\partial x^{v}}-\frac{\partial g_{v v}}{\partial x^{v}}\right) \frac{1}{2} g^{v \mu}\left(\frac{\partial \widehat{g_{v v}}}{\partial x^{v}}+\frac{\partial g_{v v}}{\partial x^{v}}-\frac{\partial g_{v v}}{\partial x^{v}}\right) \\
& \times \frac{1}{2} g^{v v}\left(\frac{\partial \widehat{g_{v v}}}{\partial x^{v}}+\frac{\partial g_{v v}}{\partial x^{v}}-\frac{\partial g_{v v}}{\partial x^{v}}\right) \\
& +\frac{\partial x^{\mu}}{\partial x^{v}} \frac{1}{2} g^{v v}\left(\frac{\partial \widehat{g_{v v}}}{\partial x^{v}}+\frac{\partial g_{v v}}{\partial x^{v}}-\frac{\partial g_{v v}}{\partial x^{v}}\right) \frac{1}{2} g^{v v}\left(\frac{\partial \widehat{g_{v v}}}{\partial x^{v}}+\frac{\partial g_{v v}}{\partial x^{v}}-\frac{\partial g_{v v}}{\partial x^{v}}\right)
\end{aligned}
$$




$$
\begin{aligned}
& +x^{v} \frac{1}{2} g^{v \mu}\left(\frac{\partial \widehat{g_{v V}}}{\partial x^{v}}+\frac{\partial g_{v v}}{\partial x^{v}}-\frac{\partial g_{v v}}{\partial x^{v}}\right) \frac{1}{2} g^{\nu v}\left(\frac{\partial \widehat{g_{v v}}}{\partial x^{v}}+\frac{\partial g_{v v}}{\partial x^{v}}-\frac{\partial g_{v v}}{\partial x^{v}}\right) \\
& \times \frac{1}{2} g^{v v}\left(\frac{\partial \widehat{g_{v v}}}{\partial x^{v}}+\frac{\partial g_{v v}}{\partial x^{v}}-\frac{\partial g_{v v}}{\partial x^{v}}\right) \\
& =\frac{\partial^{3} x^{\mu}}{\partial x^{v} \partial x^{v} \partial x^{v}}+\frac{\partial^{2}}{\partial x^{v} \partial x^{v}}\left(x^{v} \frac{1}{2}\left(\frac{\partial{\underline{g_{v}^{\mu}}}^{\nu}}{\partial x^{v}}\right)\right)+\frac{\partial}{\partial x^{v}}\left(\frac{\partial x^{v}}{\partial x^{v}} \frac{1}{2}\left(\frac{\partial \widehat{g_{v}^{\mu}}}{\partial x^{v}}\right)\right) \\
& +\frac{\partial}{\partial x^{v}}\left(x^{v} \frac{1}{2}\left(\frac{\partial \widehat{g_{v}^{v}}}{\partial x^{v}}\right) \frac{1}{2}\left(\frac{\partial{\underline{g_{v}^{\mu}}}^{\prime}}{\partial x^{v}}\right)\right)-\frac{\partial}{\partial x^{v}}\left(\frac{\partial x^{\mu}}{\partial x^{v}} \frac{1}{2}\left(\frac{\partial \widehat{g_{v}^{v}}}{\partial x^{v}}\right)\right) \\
& -\frac{\partial}{\partial x^{v}}\left(x^{v} \frac{1}{2}\left(\frac{\partial \widehat{g_{v}^{\mu}}}{\partial x^{v}}\right) \frac{1}{2}\left(\frac{\partial g_{v}^{v}}{\partial x^{v}}\right)\right)+\frac{\partial^{2} x^{v}}{\partial x^{v} \partial x^{v}} \frac{1}{2}\left(\frac{\partial \widehat{g_{v}^{\mu}}}{\partial x^{v}}\right) \\
& +\frac{\partial}{\partial x^{v}}\left(x^{v} \frac{1}{2}\left(\frac{\partial \widehat{g_{v}^{v}}}{\partial x^{v}}\right)\right) \frac{1}{2}\left(\frac{\partial \widehat{g_{v}^{\mu}}}{\partial x^{v}}\right)+\frac{\partial x^{v}}{\partial x^{v}} \frac{1}{2}\left(\frac{\partial \widehat{g_{v}^{v}}}{\partial x^{v}}\right) \frac{1}{2}\left(\frac{\partial g_{v}^{\mu}}{\partial x^{v}}\right) \\
& +x^{\nu} \frac{1}{2}\left(\frac{\partial \widehat{g_{v}^{v}}}{\partial x^{v}}\right) \frac{1}{2}\left(\frac{\partial \widehat{g_{v}^{v}}}{\partial x^{v}}\right) \frac{1}{2}\left(\frac{\partial{\underline{g_{v}^{\mu}}}_{v}}{\partial x^{v}}\right)-\frac{\partial x^{v}}{\partial x^{v}} \frac{1}{2}\left(\frac{\partial \widehat{g_{v}^{v}}}{\partial x^{v}}\right) \frac{1}{2}\left(\frac{\partial \widehat{g_{v}^{\mu}}}{\partial x^{v}}\right) \\
& -x^{\nu} \frac{1}{2}\left(\frac{\partial \widehat{g_{v}^{v}}}{\partial x^{v}}\right) \frac{1}{2}\left(\frac{\partial g_{v}^{v}}{\partial x^{v}}\right) \frac{1}{2}\left(\frac{\partial \widehat{g_{v}^{\mu}}}{\partial x^{v}}\right)-\frac{\partial^{2} x^{\mu}}{\partial x^{v} \partial x^{v}} \frac{1}{2}\left(\frac{\partial g_{v}^{v}}{\partial x^{v}}\right) \\
& -\frac{\partial}{\partial x^{v}}\left(x^{v} \frac{1}{2}\left(\frac{\partial \widehat{g_{v}^{\mu}}}{\partial x^{v}}\right)\right) \frac{1}{2}\left(\frac{\partial \widehat{g_{v}^{v}}}{\partial x^{v}}\right)-\frac{\partial x^{v}}{\partial x^{v}} \frac{1}{2}\left(\frac{\partial{g_{v}^{\mu}}_{v}}{\partial x^{v}}\right) \frac{1}{2}\left(\frac{\partial \widehat{g_{v}^{v}}}{\partial x^{v}}\right) \\
& -x^{\nu} \frac{1}{2}\left(\frac{\partial \widehat{g_{v}^{v}}}{\partial x^{v}}\right) \frac{1}{2}\left(\frac{\partial \widehat{g_{v}^{\mu}}}{\partial x^{v}}\right) \frac{1}{2}\left(\frac{\partial \widehat{g_{v}^{v}}}{\partial x^{v}}\right)+\frac{\partial x^{\mu}}{\partial x^{\nu}} \frac{1}{2}\left(\frac{\partial \widehat{g_{v}^{v}}}{\partial x^{v}}\right) \frac{1}{2}\left(\frac{\partial \widehat{g_{v}^{v}}}{\partial x^{v}}\right) \\
& +x^{v} \frac{1}{2}\left(\frac{\partial \widehat{g_{v}^{\mu}}}{\partial x^{v}}\right) \frac{1}{2}\left(\frac{\partial \widehat{g_{v}^{v}}}{\partial x^{v}}\right) \frac{1}{2}\left(\frac{\partial \widehat{g_{v}^{v}}}{\partial x^{v}}\right)-\frac{\partial^{2} x^{\mu}}{\partial x^{v} \partial x^{v}} \frac{1}{2}\left(\frac{\partial \widehat{g_{v}^{v}}}{\partial x^{v}}\right) \\
& -\frac{\partial}{\partial x^{v}}\left(x^{v} \frac{1}{2}\left(\frac{\partial \widehat{g_{v}^{\mu}}}{\partial x^{v}}\right)\right) \frac{1}{2}\left(\frac{\partial \widehat{g_{v}^{v}}}{\partial x^{v}}\right)-\frac{\partial x^{v}}{\partial x^{v}} \frac{1}{2}\left(\frac{\partial{g_{v}^{\mu}}^{\mu}}{\partial x^{v}}\right) \frac{1}{2}\left(\frac{\partial{\underline{g_{v}^{v}}}^{v}}{\partial x^{v}}\right) \\
& -x^{\nu} \frac{1}{2}\left(\frac{\partial \widehat{g_{v}^{v}}}{\partial x^{v}}\right) \frac{1}{2}\left(\frac{\partial \widehat{g_{v}^{\mu}}}{\partial x^{v}}\right) \frac{1}{2}\left(\frac{\partial \widehat{g_{v}^{v}}}{\partial x^{v}}\right)+\frac{\partial x^{\mu}}{\partial x^{\nu}} \frac{1}{2}\left(\frac{\partial \widehat{g_{v}^{v}}}{\partial x^{v}}\right) \frac{1}{2}\left(\frac{\partial \widehat{g_{v}^{v}}}{\partial x^{v}}\right) \\
& +x^{v} \frac{1}{2}\left(\frac{\partial \widehat{g_{v}^{\mu}}}{\partial x^{v}}\right) \frac{1}{2}\left(\frac{\partial{\underline{g_{v}^{v}}}^{v}}{\partial x^{v}}\right) \frac{1}{2}\left(\frac{\partial \widehat{g_{v}^{v}}}{\partial x^{v}}\right) \\
& =\frac{\partial^{3} x^{\mu}}{\partial x^{\nu} \partial x^{\nu} \partial x^{\nu}}+\frac{\partial^{2}}{\partial x^{\nu} \partial x^{\nu}}\left(x^{\nu} \frac{1}{2}\left(\frac{\partial g_{v}^{\mu}}{\partial x^{\nu}}\right)\right)+\frac{\partial}{\partial x^{v}}\left(\frac{\partial x^{\nu}}{\partial x^{\nu}} \frac{1}{2}\left(\frac{\partial g_{v}^{\mu}}{\partial x^{\nu}}\right)\right) \\
& +\frac{\partial}{\partial x^{v}}\left(x^{\nu} \frac{1}{2}\left(\frac{\partial g_{v}^{v}}{\partial x^{v}}\right) \frac{1}{2}\left(\frac{\partial g_{v}^{\mu}}{\partial x^{v}}\right)\right)-\frac{\partial}{\partial x^{v}}\left(\frac{\partial x^{\mu}}{\partial x^{v}} \frac{1}{2}\left(\frac{\partial g_{v}^{v}}{\partial x^{v}}\right)\right) \\
& -\frac{\partial}{\partial x^{v}}\left(x^{v} \frac{1}{2}\left(\frac{\partial g_{v}^{\mu}}{\partial x^{v}}\right) \frac{1}{2}\left(\frac{\partial g_{v}^{v}}{\partial x^{v}}\right)\right)+\frac{\partial^{2} x^{v}}{\partial x^{v} \partial x^{v}} \frac{1}{2}\left(\frac{\partial g_{v}^{\mu}}{\partial x^{v}}\right)
\end{aligned}
$$




$$
\begin{aligned}
& +\frac{\partial}{\partial x^{v}}\left(x^{\nu} \frac{1}{2}\left(\frac{\partial g_{v}^{v}}{\partial x^{v}}\right)\right) \frac{1}{2}\left(\frac{\partial g_{v}^{\mu}}{\partial x^{v}}\right)+\frac{\partial x^{v}}{\partial x^{v}} \frac{1}{2}\left(\frac{\partial g_{v}^{v}}{\partial x^{v}}\right) \frac{1}{2}\left(\frac{\partial g_{v}^{\mu}}{\partial x^{v}}\right) \\
& +x^{\nu} \frac{1}{2}\left(\frac{\partial g_{v}^{v}}{\partial x^{v}}\right) \frac{1}{2}\left(\frac{\partial g_{v}^{v}}{\partial x^{v}}\right) \frac{1}{2}\left(\frac{\partial g_{v}^{\mu}}{\partial x^{v}}\right)-\frac{\partial x^{\nu}}{\partial x^{\nu}} \frac{1}{2}\left(\frac{\partial g_{v}^{v}}{\partial x^{v}}\right) \frac{1}{2}\left(\frac{\partial g_{v}^{\mu}}{\partial x^{v}}\right) \\
& -x^{v} \frac{1}{2}\left(\frac{\partial g_{v}^{v}}{\partial x^{v}}\right) \frac{1}{2}\left(\frac{\partial g_{v}^{v}}{\partial x^{v}}\right) \frac{1}{2}\left(\frac{\partial g_{v}^{\mu}}{\partial x^{v}}\right)-\frac{\partial^{2} x^{\mu}}{\partial x^{v} \partial x^{v}} \frac{1}{2}\left(\frac{\partial g_{v}^{v}}{\partial x^{v}}\right) \\
& -\frac{\partial}{\partial x^{v}}\left(x^{v} \frac{1}{2}\left(\frac{\partial g_{v}^{\mu}}{\partial x^{v}}\right)\right) \frac{1}{2}\left(\frac{\partial g_{v}^{v}}{\partial x^{v}}\right)-\frac{\partial x^{v}}{\partial x^{v}} \frac{1}{2}\left(\frac{\partial g_{v}^{\mu}}{\partial x^{v}}\right) \frac{1}{2}\left(\frac{\partial g_{v}^{v}}{\partial x^{v}}\right) \\
& -x^{\nu} \frac{1}{2}\left(\frac{\partial g_{v}^{v}}{\partial x^{v}}\right) \frac{1}{2}\left(\frac{\partial g_{v}^{\mu}}{\partial x^{\nu}}\right) \frac{1}{2}\left(\frac{\partial g_{v}^{v}}{\partial x^{\nu}}\right)+\frac{\partial x^{\mu}}{\partial x^{\nu}} \frac{1}{2}\left(\frac{\partial g_{v}^{v}}{\partial x^{\nu}}\right) \frac{1}{2}\left(\frac{\partial g_{v}^{v}}{\partial x^{\nu}}\right) \\
& +x^{\nu} \frac{1}{2}\left(\frac{\partial g_{v}^{\mu}}{\partial x^{v}}\right) \frac{1}{2}\left(\frac{\partial g_{v}^{v}}{\partial x^{v}}\right) \frac{1}{2}\left(\frac{\partial g_{v}^{v}}{\partial x^{v}}\right)-\frac{\partial^{2} x^{\mu}}{\partial x^{v} \partial x^{v}} \frac{1}{2}\left(\frac{\partial g_{v}^{v}}{\partial x^{v}}\right) \\
& -\frac{\partial}{\partial x^{v}}\left(x^{\nu} \frac{1}{2}\left(\frac{\partial g_{v}^{\mu}}{\partial x^{\nu}}\right)\right) \frac{1}{2}\left(\frac{\partial g_{v}^{v}}{\partial x^{v}}\right)-\frac{\partial x^{v}}{\partial x^{v}} \frac{1}{2}\left(\frac{\partial g_{v}^{\mu}}{\partial x^{\nu}}\right) \frac{1}{2}\left(\frac{\partial g_{v}^{v}}{\partial x^{\nu}}\right) \\
& -x^{\nu} \frac{1}{2}\left(\frac{\partial g_{v}^{v}}{\partial x^{v}}\right) \frac{1}{2}\left(\frac{\partial g_{v}^{\mu}}{\partial x^{v}}\right) \frac{1}{2}\left(\frac{\partial g_{v}^{v}}{\partial x^{v}}\right)+\frac{\partial x^{\mu}}{\partial x^{v}} \frac{1}{2}\left(\frac{\partial g_{v}^{v}}{\partial x^{v}}\right) \frac{1}{2}\left(\frac{\partial g_{v}^{v}}{\partial x^{v}}\right) \\
& +x^{\nu} \frac{1}{2}\left(\frac{\partial g_{v}^{\mu}}{\partial x^{\nu}}\right) \frac{1}{2}\left(\frac{\partial g_{v}^{v}}{\partial x^{v}}\right) \frac{1}{2}\left(\frac{\partial g_{v}^{v}}{\partial x^{v}}\right)
\end{aligned}
$$

from Definition 13. (24), (25) must rewrite it in

$$
\begin{aligned}
& x_{; \nu ; ; ; \nu}^{\mu}=\frac{\partial^{3} x^{\mu}}{\partial x^{v} \partial x^{v} \partial x^{v}}+\frac{\partial^{2}}{\partial x^{\nu} \partial x^{v}}\left(x^{\sigma} \frac{1}{2}\left(\frac{\partial g_{\sigma}^{\mu}}{\partial x^{v}}\right)\right)+\frac{\partial}{\partial x^{v}}\left(\frac{\partial x^{\sigma}}{\partial x^{v}} \frac{1}{2}\left(\frac{\partial g_{\sigma}^{\mu}}{\partial x^{\nu}}\right)\right) \\
& +\frac{\partial}{\partial x^{v}}\left(x^{\sigma} \frac{1}{2}\left(\frac{\partial g_{\sigma}^{\sigma}}{\partial x^{\nu}}\right) \frac{1}{2}\left(\frac{\partial g_{\sigma}^{\mu}}{\partial x^{\nu}}\right)\right)-\frac{\partial}{\partial x^{\nu}}\left(\frac{\partial x^{\mu}}{\partial x^{\sigma}} \frac{1}{2}\left(\frac{\partial g_{v}^{\sigma}}{\partial x^{\nu}}\right)\right) \\
& -\frac{\partial}{\partial x^{v}}\left(x^{\sigma} \frac{1}{2}\left(\frac{\partial g_{\sigma}^{\mu}}{\partial x^{\sigma}}\right) \frac{1}{2}\left(\frac{\partial g_{v}^{\sigma}}{\partial x^{v}}\right)\right)+\frac{\partial^{2} x^{\sigma}}{\partial x^{v} \partial x^{v}} \frac{1}{2}\left(\frac{\partial g_{\sigma}^{\mu}}{\partial x^{v}}\right) \\
& +\frac{\partial}{\partial x^{v}}\left(x^{\sigma} \frac{1}{2}\left(\frac{\partial g_{\sigma}^{\sigma}}{\partial x^{v}}\right)\right) \frac{1}{2}\left(\frac{\partial g_{\sigma}^{\mu}}{\partial x^{\nu}}\right)+\frac{\partial x^{\sigma}}{\partial x^{\nu}} \frac{1}{2}\left(\frac{\partial g_{\sigma}^{\sigma}}{\partial x^{\nu}}\right) \frac{1}{2}\left(\frac{\partial g_{\sigma}^{\mu}}{\partial x^{\nu}}\right) \\
& +x^{\sigma} \frac{1}{2}\left(\frac{\partial g_{\sigma}^{\sigma}}{\partial x^{\nu}}\right) \frac{1}{2}\left(\frac{\partial g_{\sigma}^{\sigma}}{\partial x^{\nu}}\right) \frac{1}{2}\left(\frac{\partial g_{\sigma}^{\mu}}{\partial x^{\nu}}\right)-\frac{\partial x^{\sigma}}{\partial x^{\sigma}} \frac{1}{2}\left(\frac{\partial g_{v}^{\sigma}}{\partial x^{\nu}}\right) \frac{1}{2}\left(\frac{\partial g_{\sigma}^{\mu}}{\partial x^{\nu}}\right) \\
& -x^{\sigma} \frac{1}{2}\left(\frac{\partial g_{\sigma}^{\sigma}}{\partial x^{\sigma}}\right) \frac{1}{2}\left(\frac{\partial g_{v}^{\sigma}}{\partial x^{\nu}}\right) \frac{1}{2}\left(\frac{\partial g_{\sigma}^{\mu}}{\partial x^{\nu}}\right)-\frac{\partial^{2} x^{\mu}}{\partial x^{\sigma} \partial x^{\nu}} \frac{1}{2}\left(\frac{\partial g_{v}^{\sigma}}{\partial x^{\nu}}\right) \\
& -\frac{\partial}{\partial x^{v}}\left(x^{\sigma} \frac{1}{2}\left(\frac{\partial g_{\sigma}^{\mu}}{\partial x^{\sigma}}\right)\right) \frac{1}{2}\left(\frac{\partial g_{v}^{\sigma}}{\partial x^{\nu}}\right)-\frac{\partial x^{\sigma}}{\partial x^{\sigma}} \frac{1}{2}\left(\frac{\partial g_{\sigma}^{\mu}}{\partial x^{v}}\right) \frac{1}{2}\left(\frac{\partial g_{v}^{\sigma}}{\partial x^{v}}\right) \\
& -x^{\sigma} \frac{1}{2}\left(\frac{\partial g_{\sigma}^{\sigma}}{\partial x^{\sigma}}\right) \frac{1}{2}\left(\frac{\partial g_{\sigma}^{\mu}}{\partial x^{\nu}}\right) \frac{1}{2}\left(\frac{\partial g_{v}^{\sigma}}{\partial x^{\nu}}\right)+\frac{\partial x^{\mu}}{\partial x^{\sigma}} \frac{1}{2}\left(\frac{\partial g_{\sigma}^{\sigma}}{\partial x^{\nu}}\right) \frac{1}{2}\left(\frac{\partial g_{v}^{\sigma}}{\partial x^{\nu}}\right) \\
& +x^{\sigma} \frac{1}{2}\left(\frac{\partial g_{\sigma}^{\mu}}{\partial x^{\sigma}}\right) \frac{1}{2}\left(\frac{\partial g_{\sigma}^{\sigma}}{\partial x^{v}}\right) \frac{1}{2}\left(\frac{\partial g_{v}^{\sigma}}{\partial x^{v}}\right)-\frac{\partial^{2} x^{\mu}}{\partial x^{v} \partial x^{\sigma}} \frac{1}{2}\left(\frac{\partial g_{v}^{\sigma}}{\partial x^{v}}\right)
\end{aligned}
$$




$$
\begin{aligned}
& -\frac{\partial}{\partial x^{\sigma}}\left(x^{\sigma} \frac{1}{2}\left(\frac{\partial g_{\sigma}^{\mu}}{\partial x^{\nu}}\right)\right) \frac{1}{2}\left(\frac{\partial g_{v}^{\sigma}}{\partial x^{v}}\right)-\frac{\partial x^{\sigma}}{\partial x^{v}} \frac{1}{2}\left(\frac{\partial g_{\sigma}^{\mu}}{\partial x^{\sigma}}\right) \frac{1}{2}\left(\frac{\partial g_{v}^{\sigma}}{\partial x^{\nu}}\right) \\
& -x^{\sigma} \frac{1}{2}\left(\frac{\partial g_{\sigma}^{\sigma}}{\partial x^{v}}\right) \frac{1}{2}\left(\frac{\partial g_{\sigma}^{\mu}}{\partial x^{\sigma}}\right) \frac{1}{2}\left(\frac{\partial g_{v}^{\sigma}}{\partial x^{v}}\right)+\frac{\partial x^{\mu}}{\partial x^{\sigma}} \frac{1}{2}\left(\frac{\partial g_{v}^{\sigma}}{\partial x^{\sigma}}\right) \frac{1}{2}\left(\frac{\partial g_{v}^{\sigma}}{\partial x^{v}}\right) \\
& +x^{\sigma} \frac{1}{2}\left(\frac{\partial g_{\sigma}^{\mu}}{\partial x^{\sigma}}\right) \frac{1}{2}\left(\frac{\partial g_{v}^{\sigma}}{\partial x^{\sigma}}\right) \frac{1}{2}\left(\frac{\partial g_{v}^{\sigma}}{\partial x^{v}}\right) \\
& =\frac{\partial^{3} x^{\mu}}{\partial x^{v} \partial x^{v} \partial x^{v}}+\frac{\partial^{2}}{\partial x^{v} \partial x^{v}}\left(x^{\sigma} \frac{1}{2}\left(\frac{\partial g_{v}^{\mu}}{\partial x^{\sigma}}\right)\right)+\frac{\partial}{\partial x^{v}}\left(\frac{\partial x^{\sigma}}{\partial x^{v}} \frac{1}{2}\left(\frac{\partial g_{v}^{\mu}}{\partial x^{\sigma}}\right)\right) \\
& +\frac{\partial}{\partial x^{v}}\left(x^{\sigma} \frac{1}{2}\left(\frac{\partial g_{v}^{\sigma}}{\partial x^{\sigma}}\right) \frac{1}{2}\left(\frac{\partial g_{v}^{\mu}}{\partial x^{\sigma}}\right)\right)-\frac{\partial}{\partial x^{v}}\left(\frac{\partial x^{\mu}}{\partial x^{\sigma}} \frac{1}{2}\left(\frac{\partial g_{v}^{\sigma}}{\partial x^{v}}\right)\right) \\
& -\frac{\partial}{\partial x^{v}}\left(x^{\sigma} \frac{1}{2}\left(\frac{\partial g_{\sigma}^{\mu}}{\partial x^{\sigma}}\right) \frac{1}{2}\left(\frac{\partial g_{v}^{\sigma}}{\partial x^{v}}\right)\right)+\frac{\partial^{2} x^{\sigma}}{\partial x^{v} \partial x^{v}} \frac{1}{2}\left(\frac{\partial g_{v}^{\mu}}{\partial x^{\sigma}}\right) \\
& +\frac{\partial}{\partial x^{v}}\left(x^{\sigma} \frac{1}{2}\left(\frac{\partial g_{v}^{\sigma}}{\partial x^{\sigma}}\right)\right) \frac{1}{2}\left(\frac{\partial g_{v}^{\mu}}{\partial x^{\sigma}}\right)+\frac{\partial x^{\sigma}}{\partial x^{v}} \frac{1}{2}\left(\frac{\partial g_{v}^{\sigma}}{\partial x^{\sigma}}\right) \frac{1}{2}\left(\frac{\partial g_{v}^{\mu}}{\partial x^{\sigma}}\right) \\
& +x^{\sigma} \frac{1}{2}\left(\frac{\partial g_{v}^{\sigma}}{\partial x^{\sigma}}\right) \frac{1}{2}\left(\frac{\partial g_{v}^{\sigma}}{\partial x^{\sigma}}\right) \frac{1}{2}\left(\frac{\partial g_{v}^{\mu}}{\partial x^{\sigma}}\right)-\frac{\partial x^{\sigma}}{\partial x^{\sigma}} \frac{1}{2}\left(\frac{\partial g_{v}^{\sigma}}{\partial x^{v}}\right) \frac{1}{2}\left(\frac{\partial g_{v}^{\mu}}{\partial x^{\sigma}}\right) \\
& -x^{\sigma} \frac{1}{2}\left(\frac{\partial g_{\sigma}^{\sigma}}{\partial x^{\sigma}}\right) \frac{1}{2}\left(\frac{\partial g_{v}^{\sigma}}{\partial x^{v}}\right) \frac{1}{2}\left(\frac{\partial g_{v}^{\mu}}{\partial x^{\sigma}}\right)-\frac{\partial^{2} x^{\mu}}{\partial x^{\sigma} \partial x^{\nu}} \frac{1}{2}\left(\frac{\partial g_{v}^{\sigma}}{\partial x^{\nu}}\right) \\
& -\frac{\partial}{\partial x^{v}}\left(x^{\sigma} \frac{1}{2}\left(\frac{\partial g_{\sigma}^{\mu}}{\partial x^{\sigma}}\right)\right) \frac{1}{2}\left(\frac{\partial g_{v}^{\sigma}}{\partial x^{v}}\right)-\frac{\partial x^{\sigma}}{\partial x^{\sigma}} \frac{1}{2}\left(\frac{\partial g_{v}^{\mu}}{\partial x^{\sigma}}\right) \frac{1}{2}\left(\frac{\partial g_{v}^{\sigma}}{\partial x^{v}}\right) \\
& -x^{\sigma} \frac{1}{2}\left(\frac{\partial g_{\sigma}^{\sigma}}{\partial x^{\sigma}}\right) \frac{1}{2}\left(\frac{\partial g_{v}^{\mu}}{\partial x^{\sigma}}\right) \frac{1}{2}\left(\frac{\partial g_{v}^{\sigma}}{\partial x^{v}}\right)+\frac{\partial x^{\mu}}{\partial x^{\sigma}} \frac{1}{2}\left(\frac{\partial g_{v}^{\sigma}}{\partial x^{\sigma}}\right) \frac{1}{2}\left(\frac{\partial g_{v}^{\sigma}}{\partial x^{v}}\right) \\
& +x^{\sigma} \frac{1}{2}\left(\frac{\partial g_{\sigma}^{\mu}}{\partial x^{\sigma}}\right) \frac{1}{2}\left(\frac{\partial g_{v}^{\sigma}}{\partial x^{\sigma}}\right) \frac{1}{2}\left(\frac{\partial g_{v}^{\sigma}}{\partial x^{v}}\right)-\frac{\partial^{2} x^{\mu}}{\partial x^{v} \partial x^{\sigma}} \frac{1}{2}\left(\frac{\partial g_{v}^{\sigma}}{\partial x^{v}}\right) \\
& -\frac{\partial}{\partial x^{\sigma}}\left(x^{\sigma} \frac{1}{2}\left(\frac{\partial g_{v}^{\mu}}{\partial x^{\sigma}}\right)\right) \frac{1}{2}\left(\frac{\partial g_{v}^{\sigma}}{\partial x^{v}}\right)-\frac{\partial x^{\sigma}}{\partial x^{v}} \frac{1}{2}\left(\frac{\partial g_{\sigma}^{\mu}}{\partial x^{\sigma}}\right) \frac{1}{2}\left(\frac{\partial g_{v}^{\sigma}}{\partial x^{v}}\right) \\
& -x^{\sigma} \frac{1}{2}\left(\frac{\partial g_{v}^{\sigma}}{\partial x^{\sigma}}\right) \frac{1}{2}\left(\frac{\partial g_{\sigma}^{\mu}}{\partial x^{\sigma}}\right) \frac{1}{2}\left(\frac{\partial g_{v}^{\sigma}}{\partial x^{v}}\right)+\frac{\partial x^{\mu}}{\partial x^{\sigma}} \frac{1}{2}\left(\frac{\partial g_{\sigma}^{\sigma}}{\partial x^{v}}\right) \frac{1}{2}\left(\frac{\partial g_{v}^{\sigma}}{\partial x^{v}}\right) \\
& +x^{\sigma} \frac{1}{2}\left(\frac{\partial g_{\sigma}^{\mu}}{\partial x^{\sigma}}\right) \frac{1}{2}\left(\frac{\partial g_{\sigma}^{\sigma}}{\partial x^{v}}\right) \frac{1}{2}\left(\frac{\partial g_{v}^{\sigma}}{\partial x^{v}}\right)
\end{aligned}
$$

by (24), (25) being a tensor equation. The dummy index has an invariable property for consideration of Binary Law. In other words, the index which was dummy index in Definition 13 is dummy index in (26), (27). I get the conclusion that (26), (27) doesn't satisfy Binary Law from Definition 6. I get the conclusion that Definition 13 isn't an equation of the tensor satisfying Binary Law because (26), (27) doesn't satisfy Binary Law.

I rewrite three existing index $v$ in each term of (26), (27) in index $\mu$ using Definition 4 and get 


$$
\begin{aligned}
& -x_{; \mu ; \mu ; \mu}^{\mu}=-\frac{\partial^{3} x^{\mu}}{\partial x^{\mu} \partial x^{\mu} \partial x^{\mu}}-\frac{\partial^{2}}{\partial x^{\mu} \partial x^{\mu}}\left(x^{\sigma} \frac{1}{2}\left(\frac{\partial g_{\sigma}^{\mu}}{\partial x^{\mu}}\right)\right)-\frac{\partial}{\partial x^{\mu}}\left(\frac{\partial x^{\sigma}}{\partial x^{\mu}} \frac{1}{2}\left(\frac{\partial g_{\sigma}^{\mu}}{\partial x^{\mu}}\right)\right) \\
& -\frac{\partial}{\partial x^{\mu}}\left(x^{\sigma} \frac{1}{2}\left(\frac{\partial g_{\sigma}^{\sigma}}{\partial x^{\mu}}\right) \frac{1}{2}\left(\frac{\partial g_{\sigma}^{\mu}}{\partial x^{\mu}}\right)\right)+\frac{\partial}{\partial x^{\mu}}\left(\frac{\partial x^{\mu}}{\partial x^{\sigma}} \frac{1}{2}\left(\frac{\partial g_{\mu}^{\sigma}}{\partial x^{\mu}}\right)\right) \\
& +\frac{\partial}{\partial x^{\mu}}\left(x^{\sigma} \frac{1}{2}\left(\frac{\partial g_{\sigma}^{\mu}}{\partial x^{\sigma}}\right) \frac{1}{2}\left(\frac{\partial g_{\mu}^{\sigma}}{\partial x^{\mu}}\right)\right)-\frac{\partial^{2} x^{\sigma}}{\partial x^{\mu} \partial x^{\mu}} \frac{1}{2}\left(\frac{\partial g_{\sigma}^{\mu}}{\partial x^{\mu}}\right) \\
& -\frac{\partial}{\partial x^{\mu}}\left(x^{\sigma} \frac{1}{2}\left(\frac{\partial g_{\sigma}^{\sigma}}{\partial x^{\mu}}\right)\right) \frac{1}{2}\left(\frac{\partial g_{\sigma}^{\mu}}{\partial x^{\mu}}\right)-\frac{\partial x^{\sigma}}{\partial x^{\mu}} \frac{1}{2}\left(\frac{\partial g_{\sigma}^{\sigma}}{\partial x^{\mu}}\right) \frac{1}{2}\left(\frac{\partial g_{\sigma}^{\mu}}{\partial x^{\mu}}\right) \\
& -x^{\sigma} \frac{1}{2}\left(\frac{\partial g_{\sigma}^{\sigma}}{\partial x^{\mu}}\right) \frac{1}{2}\left(\frac{\partial g_{\sigma}^{\sigma}}{\partial x^{\mu}}\right) \frac{1}{2}\left(\frac{\partial g_{\sigma}^{\mu}}{\partial x^{\mu}}\right)+\frac{\partial x^{\sigma}}{\partial x^{\sigma}} \frac{1}{2}\left(\frac{\partial g_{\mu}^{\sigma}}{\partial x^{\mu}}\right) \frac{1}{2}\left(\frac{\partial g_{\sigma}^{\mu}}{\partial x^{\mu}}\right) \\
& +x^{\sigma} \frac{1}{2}\left(\frac{\partial g_{\sigma}^{\sigma}}{\partial x^{\sigma}}\right) \frac{1}{2}\left(\frac{\partial g_{\mu}^{\sigma}}{\partial x^{\mu}}\right) \frac{1}{2}\left(\frac{\partial g_{\sigma}^{\mu}}{\partial x^{\mu}}\right)+\frac{\partial^{2} x^{\mu}}{\partial x^{\sigma} \partial x^{\mu}} \frac{1}{2}\left(\frac{\partial g_{\mu}^{\sigma}}{\partial x^{\mu}}\right) \\
& +\frac{\partial}{\partial x^{\mu}}\left(x^{\sigma} \frac{1}{2}\left(\frac{\partial g_{\sigma}^{\mu}}{\partial x^{\sigma}}\right)\right) \frac{1}{2}\left(\frac{\partial g_{\mu}^{\sigma}}{\partial x^{\mu}}\right)+\frac{\partial x^{\sigma}}{\partial x^{\sigma}} \frac{1}{2}\left(\frac{\partial g_{\sigma}^{\mu}}{\partial x^{\mu}}\right) \frac{1}{2}\left(\frac{\partial g_{\mu}^{\sigma}}{\partial x^{\mu}}\right) \\
& +x^{\sigma} \frac{1}{2}\left(\frac{\partial g_{\sigma}^{\sigma}}{\partial x^{\sigma}}\right) \frac{1}{2}\left(\frac{\partial g_{\sigma}^{\mu}}{\partial x^{\mu}}\right) \frac{1}{2}\left(\frac{\partial g_{\mu}^{\sigma}}{\partial x^{\mu}}\right)-\frac{\partial x^{\mu}}{\partial x^{\sigma}} \frac{1}{2}\left(\frac{\partial g_{\sigma}^{\sigma}}{\partial x^{\mu}}\right) \frac{1}{2}\left(\frac{\partial g_{\mu}^{\sigma}}{\partial x^{\mu}}\right) \\
& -x^{\sigma} \frac{1}{2}\left(\frac{\partial g_{\sigma}^{\mu}}{\partial x^{\sigma}}\right) \frac{1}{2}\left(\frac{\partial g_{\sigma}^{\sigma}}{\partial x^{\mu}}\right) \frac{1}{2}\left(\frac{\partial g_{\mu}^{\sigma}}{\partial x^{\mu}}\right)+\frac{\partial^{2} x^{\mu}}{\partial x^{\mu} \partial x^{\sigma}} \frac{1}{2}\left(\frac{\partial g_{\mu}^{\sigma}}{\partial x^{\mu}}\right) \\
& +\frac{\partial}{\partial x^{\sigma}}\left(x^{\sigma} \frac{1}{2}\left(\frac{\partial g_{\sigma}^{\mu}}{\partial x^{\mu}}\right)\right) \frac{1}{2}\left(\frac{\partial g_{\mu}^{\sigma}}{\partial x^{\mu}}\right)+\frac{\partial x^{\sigma}}{\partial x^{\mu}} \frac{1}{2}\left(\frac{\partial g_{\sigma}^{\mu}}{\partial x^{\sigma}}\right) \frac{1}{2}\left(\frac{\partial g_{\mu}^{\sigma}}{\partial x^{\mu}}\right) \\
& +x^{\sigma} \frac{1}{2}\left(\frac{\partial g_{\sigma}^{\sigma}}{\partial x^{\mu}}\right) \frac{1}{2}\left(\frac{\partial g_{\sigma}^{\mu}}{\partial x^{\sigma}}\right) \frac{1}{2}\left(\frac{\partial g_{\mu}^{\sigma}}{\partial x^{\mu}}\right)-\frac{\partial x^{\mu}}{\partial x^{\sigma}} \frac{1}{2}\left(\frac{\partial g_{\mu}^{\sigma}}{\partial x^{\sigma}}\right) \frac{1}{2}\left(\frac{\partial g_{\mu}^{\sigma}}{\partial x^{\mu}}\right) \\
& -x^{\sigma} \frac{1}{2}\left(\frac{\partial g_{\sigma}^{\mu}}{\partial x^{\sigma}}\right) \frac{1}{2}\left(\frac{\partial g_{\mu}^{\sigma}}{\partial x^{\sigma}}\right) \frac{1}{2}\left(\frac{\partial g_{\mu}^{\sigma}}{\partial x^{\mu}}\right) \\
& =-\frac{\partial^{3} x^{\mu}}{\partial x^{\mu} \partial x^{\mu} \partial x^{\mu}}-\frac{\partial^{2}}{\partial x^{\mu} \partial x^{\mu}}\left(x^{\sigma} \frac{1}{2}\left(\frac{\partial g_{\mu}^{\mu}}{\partial x^{\sigma}}\right)\right)-\frac{\partial}{\partial x^{\mu}}\left(\frac{\partial x^{\sigma}}{\partial x^{\mu}} \frac{1}{2}\left(\frac{\partial g_{\mu}^{\mu}}{\partial x^{\sigma}}\right)\right) \\
& -\frac{\partial}{\partial x^{\mu}}\left(x^{\sigma} \frac{1}{2}\left(\frac{\partial g_{\mu}^{\sigma}}{\partial x^{\sigma}}\right) \frac{1}{2}\left(\frac{\partial g_{\mu}^{\mu}}{\partial x^{\sigma}}\right)\right)+\frac{\partial}{\partial x^{\mu}}\left(\frac{\partial x^{\mu}}{\partial x^{\sigma}} \frac{1}{2}\left(\frac{\partial g_{\mu}^{\sigma}}{\partial x^{\mu}}\right)\right) \\
& +\frac{\partial}{\partial x^{\mu}}\left(x^{\sigma} \frac{1}{2}\left(\frac{\partial g_{\sigma}^{\mu}}{\partial x^{\sigma}}\right) \frac{1}{2}\left(\frac{\partial g_{\mu}^{\sigma}}{\partial x^{\mu}}\right)\right)-\frac{\partial^{2} x^{\sigma}}{\partial x^{\mu} \partial x^{\mu}} \frac{1}{2}\left(\frac{\partial g_{\mu}^{\mu}}{\partial x^{\sigma}}\right) \\
& -\frac{\partial}{\partial x^{\mu}}\left(x^{\sigma} \frac{1}{2}\left(\frac{\partial g_{\mu}^{\sigma}}{\partial x^{\sigma}}\right)\right) \frac{1}{2}\left(\frac{\partial g_{\mu}^{\mu}}{\partial x^{\sigma}}\right)-\frac{\partial x^{\sigma}}{\partial x^{\mu}} \frac{1}{2}\left(\frac{\partial g_{\mu}^{\sigma}}{\partial x^{\sigma}}\right) \frac{1}{2}\left(\frac{\partial g_{\mu}^{\mu}}{\partial x^{\sigma}}\right) \\
& -x^{\sigma} \frac{1}{2}\left(\frac{\partial g_{\mu}^{\sigma}}{\partial x^{\sigma}}\right) \frac{1}{2}\left(\frac{\partial g_{\mu}^{\sigma}}{\partial x^{\sigma}}\right) \frac{1}{2}\left(\frac{\partial g_{\mu}^{\mu}}{\partial x^{\sigma}}\right)+\frac{\partial x^{\sigma}}{\partial x^{\sigma}} \frac{1}{2}\left(\frac{\partial g_{\mu}^{\sigma}}{\partial x^{\mu}}\right) \frac{1}{2}\left(\frac{\partial g_{\mu}^{\mu}}{\partial x^{\sigma}}\right) \\
& +x^{\sigma} \frac{1}{2}\left(\frac{\partial g_{\sigma}^{\sigma}}{\partial x^{\sigma}}\right) \frac{1}{2}\left(\frac{\partial g_{\mu}^{\sigma}}{\partial x^{\mu}}\right) \frac{1}{2}\left(\frac{\partial g_{\mu}^{\mu}}{\partial x^{\sigma}}\right)+\frac{\partial^{2} x^{\mu}}{\partial x^{\sigma} \partial x^{\mu}} \frac{1}{2}\left(\frac{\partial g_{\mu}^{\sigma}}{\partial x^{\mu}}\right)
\end{aligned}
$$




$$
\begin{aligned}
& +\frac{\partial}{\partial x^{\mu}}\left(x^{\sigma} \frac{1}{2}\left(\frac{\partial g_{\sigma}^{\mu}}{\partial x^{\sigma}}\right)\right) \frac{1}{2}\left(\frac{\partial g_{\mu}^{\sigma}}{\partial x^{\mu}}\right)+\frac{\partial x^{\sigma}}{\partial x^{\sigma}} \frac{1}{2}\left(\frac{\partial g_{\mu}^{\mu}}{\partial x^{\sigma}}\right) \frac{1}{2}\left(\frac{\partial g_{\mu}^{\sigma}}{\partial x^{\mu}}\right) \\
& +x^{\sigma} \frac{1}{2}\left(\frac{\partial g_{\sigma}^{\sigma}}{\partial x^{\sigma}}\right) \frac{1}{2}\left(\frac{\partial g_{\mu}^{\mu}}{\partial x^{\sigma}}\right) \frac{1}{2}\left(\frac{\partial g_{\mu}^{\sigma}}{\partial x^{\mu}}\right)-\frac{\partial x^{\mu}}{\partial x^{\sigma}} \frac{1}{2}\left(\frac{\partial g_{\mu}^{\sigma}}{\partial x^{\sigma}}\right) \frac{1}{2}\left(\frac{\partial g_{\mu}^{\sigma}}{\partial x^{\mu}}\right) \\
& -x^{\sigma} \frac{1}{2}\left(\frac{\partial g_{\sigma}^{\mu}}{\partial x^{\sigma}}\right) \frac{1}{2}\left(\frac{\partial g_{\mu}^{\sigma}}{\partial x^{\sigma}}\right) \frac{1}{2}\left(\frac{\partial g_{\mu}^{\sigma}}{\partial x^{\mu}}\right)+\frac{\partial^{2} x^{\mu}}{\partial x^{\mu} \partial x^{\sigma}} \frac{1}{2}\left(\frac{\partial g_{\mu}^{\sigma}}{\partial x^{\mu}}\right) \\
& +\frac{\partial}{\partial x^{\sigma}}\left(x^{\sigma} \frac{1}{2}\left(\frac{\partial g_{\mu}^{\mu}}{\partial x^{\sigma}}\right)\right) \frac{1}{2}\left(\frac{\partial g_{\mu}^{\sigma}}{\partial x^{\mu}}\right)+\frac{\partial x^{\sigma}}{\partial x^{\mu}} \frac{1}{2}\left(\frac{\partial g_{\sigma}^{\mu}}{\partial x^{\sigma}}\right) \frac{1}{2}\left(\frac{\partial g_{\mu}^{\sigma}}{\partial x^{\mu}}\right) \\
& +x^{\sigma} \frac{1}{2}\left(\frac{\partial g_{\mu}^{\sigma}}{\partial x^{\sigma}}\right) \frac{1}{2}\left(\frac{\partial g_{\sigma}^{\mu}}{\partial x^{\sigma}}\right) \frac{1}{2}\left(\frac{\partial g_{\mu}^{\sigma}}{\partial x^{\mu}}\right)-\frac{\partial x^{\mu}}{\partial x^{\sigma}} \frac{1}{2}\left(\frac{\partial g_{\sigma}^{\sigma}}{\partial x^{\mu}}\right) \frac{1}{2}\left(\frac{\partial g_{\mu}^{\sigma}}{\partial x^{\mu}}\right) \\
& -x^{\sigma} \frac{1}{2}\left(\frac{\partial g_{\sigma}^{\mu}}{\partial x^{\sigma}}\right) \frac{1}{2}\left(\frac{\partial g_{\sigma}^{\sigma}}{\partial x^{\mu}}\right) \frac{1}{2}\left(\frac{\partial g_{\mu}^{\sigma}}{\partial x^{\mu}}\right) .
\end{aligned}
$$

I get

$$
-x_{; \mu ; \mu ; \mu}^{\mu}=-\frac{\partial^{3} x^{\mu}}{\partial x^{\mu} \partial x^{\mu} \partial x^{\mu}}
$$

in consideration of Definition 7 for (28). Because the second term of the right side of (29) doesn't exist,

$$
x_{;, v ; v v}^{\mu}=\frac{\partial^{3} x^{\mu}}{\partial x^{v} \partial x^{v} \partial x^{v}}
$$

can rewrite (29) using Definition 4. I rewrite three existing index $v$ in each term of (26), (27) in index $\mu$ using Definition 2 and get

$$
\begin{aligned}
x^{\mu ; \mu ; \mu ; \mu}= & \frac{\partial^{3} x^{\mu}}{\partial x_{\mu} \partial x_{\mu} \partial x_{\mu}}+\frac{\partial^{2}}{\partial x_{\mu} \partial x_{\mu}}\left(x^{\sigma} \frac{1}{2}\left(\frac{\partial g_{\sigma}^{\mu}}{\partial x_{\mu}}\right)\right)+\frac{\partial}{\partial x_{\mu}}\left(\frac{\partial x^{\sigma}}{\partial x_{\mu}} \frac{1}{2}\left(\frac{\partial g_{\sigma}^{\mu}}{\partial x_{\mu}}\right)\right) \\
& +\frac{\partial}{\partial x_{\mu}}\left(x^{\sigma} \frac{1}{2}\left(\frac{\partial g_{\sigma}^{\sigma}}{\partial x_{\mu}}\right) \frac{1}{2}\left(\frac{\partial g_{\sigma}^{\mu}}{\partial x_{\mu}}\right)\right)-\frac{\partial}{\partial x_{\mu}}\left(\frac{\partial x^{\mu}}{\partial x^{\sigma}} \frac{1}{2}\left(\frac{\partial g^{\sigma \mu}}{\partial x_{\mu}}\right)\right) \\
& -\frac{\partial}{\partial x_{\mu}}\left(x^{\sigma} \frac{1}{2}\left(\frac{\partial g_{\sigma}^{\mu}}{\partial x^{\sigma}}\right) \frac{1}{2}\left(\frac{\partial g^{\sigma \mu}}{\partial x_{\mu}}\right)\right)+\frac{\partial^{2} x^{\sigma}}{\partial x_{\mu} \partial x_{\mu}} \frac{1}{2}\left(\frac{\partial g_{\sigma}^{\mu}}{\partial x_{\mu}}\right) \\
& +\frac{\partial}{\partial x_{\mu}}\left(x^{\sigma} \frac{1}{2}\left(\frac{\partial g_{\sigma}^{\sigma}}{\partial x_{\mu}}\right)\right) \frac{1}{2}\left(\frac{\partial g_{\sigma}^{\mu}}{\partial x_{\mu}}\right)+\frac{\partial x^{\sigma}}{\partial x_{\mu}} \frac{1}{2}\left(\frac{\partial g_{\sigma}^{\sigma}}{\partial x_{\mu}}\right) \frac{1}{2}\left(\frac{\partial g_{\sigma}^{\mu}}{\partial x_{\mu}}\right) \\
& +x^{\sigma} \frac{1}{2}\left(\frac{\partial g_{\sigma}^{\sigma}}{\partial x_{\mu}}\right) \frac{1}{2}\left(\frac{\partial g_{\sigma}^{\sigma}}{\partial x_{\mu}}\right) \frac{1}{2}\left(\frac{\partial g_{\sigma}^{\mu}}{\partial x_{\mu}}\right)-\frac{\partial x^{\sigma}}{\partial x^{\sigma}} \frac{1}{2}\left(\frac{\partial g^{\sigma \mu}}{\partial x_{\mu}}\right) \frac{1}{2}\left(\frac{\partial g_{\sigma}^{\mu}}{\partial x_{\mu}}\right) \\
& -x^{\sigma} \frac{1}{2}\left(\frac{\partial g_{\sigma}^{\sigma}}{\partial x^{\sigma}}\right) \frac{1}{2}\left(\frac{\partial g^{\sigma \mu}}{\partial x_{\mu}}\right) \frac{1}{2}\left(\frac{\partial g_{\sigma}^{\mu}}{\partial x_{\mu}}\right)-\frac{\partial^{2} x^{\mu}}{\partial x^{\sigma} \partial x_{\mu}} \frac{1}{2}\left(\frac{\partial g^{\sigma \mu}}{\partial x_{\mu}}\right) \\
& -\frac{\partial}{\partial x_{\mu}}\left(x^{\sigma} \frac{1}{2}\left(\frac{\partial g_{\sigma}^{\mu}}{\partial x^{\sigma}}\right)\right) \frac{1}{2}\left(\frac{\partial g^{\sigma \mu}}{\partial x_{\mu}}\right)-\frac{\partial x^{\sigma}}{\partial x^{\sigma}} \frac{1}{2}\left(\frac{\partial g_{\sigma}^{\mu}}{\partial x_{\mu}}\right) \frac{1}{2}\left(\frac{\partial g^{\sigma \mu}}{\partial x_{\mu}}\right) \\
& -x^{\sigma} \frac{1}{2}\left(\frac{\partial g_{\sigma}^{\sigma}}{\partial x^{\sigma}}\right) \frac{1}{2}\left(\frac{\partial g_{\sigma}^{\mu}}{\partial x_{\mu}}\right) \frac{1}{2}\left(\frac{\partial g^{\sigma \mu}}{\partial x_{\mu}}\right)+\frac{\partial x^{\mu}}{\partial x^{\sigma}} \frac{1}{2}\left(\frac{\partial g_{\sigma}^{\sigma}}{\partial x_{\mu}}\right) \frac{1}{2}\left(\frac{\partial g^{\sigma \mu}}{\partial x_{\mu}}\right)
\end{aligned}
$$




$$
\begin{aligned}
& +x^{\sigma} \frac{1}{2}\left(\frac{\partial g_{\sigma}^{\mu}}{\partial x^{\sigma}}\right) \frac{1}{2}\left(\frac{\partial g_{\sigma}^{\sigma}}{\partial x_{\mu}}\right) \frac{1}{2}\left(\frac{\partial g^{\sigma \mu}}{\partial x_{\mu}}\right)-\frac{\partial^{2} x^{\mu}}{\partial x_{\mu} \partial x^{\sigma}} \frac{1}{2}\left(\frac{\partial g^{\sigma \mu}}{\partial x_{\mu}}\right) \\
& -\frac{\partial}{\partial x^{\sigma}}\left(x^{\sigma} \frac{1}{2}\left(\frac{\partial g_{\sigma}^{\mu}}{\partial x_{\mu}}\right)\right) \frac{1}{2}\left(\frac{\partial g^{\sigma \mu}}{\partial x_{\mu}}\right)-\frac{\partial x^{\sigma}}{\partial x_{\mu}} \frac{1}{2}\left(\frac{\partial g_{\sigma}^{\mu}}{\partial x^{\sigma}}\right) \frac{1}{2}\left(\frac{\partial g^{\sigma \mu}}{\partial x_{\mu}}\right) \\
& -x^{\sigma} \frac{1}{2}\left(\frac{\partial g_{\sigma}^{\sigma}}{\partial x_{\mu}}\right) \frac{1}{2}\left(\frac{\partial g_{\sigma}^{\mu}}{\partial x^{\sigma}}\right) \frac{1}{2}\left(\frac{\partial g^{\sigma \mu}}{\partial x_{\mu}}\right)+\frac{\partial x^{\mu}}{\partial x^{\sigma}} \frac{1}{2}\left(\frac{\partial g^{\sigma \mu}}{\partial x^{\sigma}}\right) \frac{1}{2}\left(\frac{\partial g^{\sigma \mu}}{\partial x_{\mu}}\right) \\
& +x^{\sigma} \frac{1}{2}\left(\frac{\partial g_{\sigma}^{\mu}}{\partial x^{\sigma}}\right) \frac{1}{2}\left(\frac{\partial g^{\sigma \mu}}{\partial x^{\sigma}}\right) \frac{1}{2}\left(\frac{\partial g^{\sigma \mu}}{\partial x_{\mu}}\right) \\
& =\frac{\partial^{3} x^{\mu}}{\partial x_{\mu} \partial x_{\mu} \partial x_{\mu}}+\frac{\partial^{2}}{\partial x_{\mu} \partial x_{\mu}}\left(x^{\sigma} \frac{1}{2}\left(\frac{\partial g^{\mu \mu}}{\partial x^{\sigma}}\right)\right)+\frac{\partial}{\partial x_{\mu}}\left(\frac{\partial x^{\sigma}}{\partial x_{\mu}} \frac{1}{2}\left(\frac{\partial g^{\mu \mu}}{\partial x^{\sigma}}\right)\right) \\
& +\frac{\partial}{\partial x_{\mu}}\left(x^{\sigma} \frac{1}{2}\left(\frac{\partial g^{\sigma \mu}}{\partial x^{\sigma}}\right) \frac{1}{2}\left(\frac{\partial g^{\mu \mu}}{\partial x^{\sigma}}\right)\right)-\frac{\partial}{\partial x_{\mu}}\left(\frac{\partial x^{\mu}}{\partial x^{\sigma}} \frac{1}{2}\left(\frac{\partial g^{\sigma \mu}}{\partial x_{\mu}}\right)\right) \\
& -\frac{\partial}{\partial x_{\mu}}\left(x^{\sigma} \frac{1}{2}\left(\frac{\partial g_{\sigma}^{\mu}}{\partial x^{\sigma}}\right) \frac{1}{2}\left(\frac{\partial g^{\sigma \mu}}{\partial x_{\mu}}\right)\right)+\frac{\partial^{2} x^{\sigma}}{\partial x_{\mu} \partial x_{\mu}} \frac{1}{2}\left(\frac{\partial g^{\mu \mu}}{\partial x^{\sigma}}\right) \\
& +\frac{\partial}{\partial x_{\mu}}\left(x^{\sigma} \frac{1}{2}\left(\frac{\partial g^{\sigma \mu}}{\partial x^{\sigma}}\right)\right) \frac{1}{2}\left(\frac{\partial g^{\mu \mu}}{\partial x^{\sigma}}\right)+\frac{\partial x^{\sigma}}{\partial x_{\mu}} \frac{1}{2}\left(\frac{\partial g^{\sigma \mu}}{\partial x^{\sigma}}\right) \frac{1}{2}\left(\frac{\partial g^{\mu \mu}}{\partial x^{\sigma}}\right) \\
& +x^{\sigma} \frac{1}{2}\left(\frac{\partial g^{\sigma \mu}}{\partial x^{\sigma}}\right) \frac{1}{2}\left(\frac{\partial g^{\sigma \mu}}{\partial x^{\sigma}}\right) \frac{1}{2}\left(\frac{\partial g^{\mu \mu}}{\partial x^{\sigma}}\right)-\frac{\partial x^{\sigma}}{\partial x^{\sigma}} \frac{1}{2}\left(\frac{\partial g^{\sigma \mu}}{\partial x_{\mu}}\right) \frac{1}{2}\left(\frac{\partial g^{\mu \mu}}{\partial x^{\sigma}}\right) \\
& -x^{\sigma} \frac{1}{2}\left(\frac{\partial g_{\sigma}^{\sigma}}{\partial x^{\sigma}}\right) \frac{1}{2}\left(\frac{\partial g^{\sigma \mu}}{\partial x_{\mu}}\right) \frac{1}{2}\left(\frac{\partial g^{\mu \mu}}{\partial x^{\sigma}}\right)-\frac{\partial^{2} x^{\mu}}{\partial x^{\sigma} \partial x_{\mu}} \frac{1}{2}\left(\frac{\partial g^{\sigma \mu}}{\partial x_{\mu}}\right) \\
& -\frac{\partial}{\partial x_{\mu}}\left(x^{\sigma} \frac{1}{2}\left(\frac{\partial g_{\sigma}^{\mu}}{\partial x^{\sigma}}\right)\right) \frac{1}{2}\left(\frac{\partial g^{\sigma \mu}}{\partial x_{\mu}}\right)-\frac{\partial x^{\sigma}}{\partial x^{\sigma}} \frac{1}{2}\left(\frac{\partial g^{\mu \mu}}{\partial x^{\sigma}}\right) \frac{1}{2}\left(\frac{\partial g^{\sigma \mu}}{\partial x_{\mu}}\right) \\
& -x^{\sigma} \frac{1}{2}\left(\frac{\partial g_{\sigma}^{\sigma}}{\partial x^{\sigma}}\right) \frac{1}{2}\left(\frac{\partial g^{\mu \mu}}{\partial x^{\sigma}}\right) \frac{1}{2}\left(\frac{\partial g^{\sigma \mu}}{\partial x_{\mu}}\right)+\frac{\partial x^{\mu}}{\partial x^{\sigma}} \frac{1}{2}\left(\frac{\partial g^{\sigma \mu}}{\partial x^{\sigma}}\right) \frac{1}{2}\left(\frac{\partial g^{\sigma \mu}}{\partial x_{\mu}}\right) \\
& +x^{\sigma} \frac{1}{2}\left(\frac{\partial g_{\sigma}^{\mu}}{\partial x^{\sigma}}\right) \frac{1}{2}\left(\frac{\partial g^{\sigma \mu}}{\partial x^{\sigma}}\right) \frac{1}{2}\left(\frac{\partial g^{\sigma \mu}}{\partial x_{\mu}}\right)-\frac{\partial^{2} x^{\mu}}{\partial x_{\mu} \partial x^{\sigma}} \frac{1}{2}\left(\frac{\partial g^{\sigma \mu}}{\partial x_{\mu}}\right) \\
& -\frac{\partial}{\partial x^{\sigma}}\left(x^{\sigma} \frac{1}{2}\left(\frac{\partial g^{\mu \mu}}{\partial x^{\sigma}}\right)\right) \frac{1}{2}\left(\frac{\partial g^{\sigma \mu}}{\partial x_{\mu}}\right)-\frac{\partial x^{\sigma}}{\partial x_{\mu}} \frac{1}{2}\left(\frac{\partial g_{\sigma}^{\mu}}{\partial x^{\sigma}}\right) \frac{1}{2}\left(\frac{\partial g^{\sigma \mu}}{\partial x_{\mu}}\right) \\
& -x^{\sigma} \frac{1}{2}\left(\frac{\partial g^{\sigma \mu}}{\partial x^{\sigma}}\right) \frac{1}{2}\left(\frac{\partial g_{\sigma}^{\mu}}{\partial x^{\sigma}}\right) \frac{1}{2}\left(\frac{\partial g^{\sigma \mu}}{\partial x_{\mu}}\right)+\frac{\partial x^{\mu}}{\partial x^{\sigma}} \frac{1}{2}\left(\frac{\partial g_{\sigma}^{\sigma}}{\partial x_{\mu}}\right) \frac{1}{2}\left(\frac{\partial g^{\sigma \mu}}{\partial x_{\mu}}\right) \\
& +x^{\sigma} \frac{1}{2}\left(\frac{\partial g_{\sigma}^{\mu}}{\partial x^{\sigma}}\right) \frac{1}{2}\left(\frac{\partial g_{\sigma}^{\sigma}}{\partial x_{\mu}}\right) \frac{1}{2}\left(\frac{\partial g^{\sigma \mu}}{\partial x_{\mu}}\right) \text {. }
\end{aligned}
$$

-End Proof-

\section{About a Coordinate Transformations Equation in Tensor Satisfying Binary Law}

Proposition 8 When all coordinate systems satisfy Binary Law, 
$x_{\mu}^{\mu}=\frac{\partial x^{\mu}}{\partial x^{\nu}} \frac{\partial x^{\nu}}{\partial x^{\mu}} x_{v}^{\nu}=x_{v}^{v}$ is established for $x_{\mu}^{\mu}$ components of a tensor satisfying Binary law of rank zero.

Proof: When all coordinate systems satisfy Binary Law, I get

$$
x_{\mu}^{\mu}=\frac{\partial x^{\mu}}{\partial x^{v}} \frac{\partial x^{v}}{\partial x^{\mu}} x_{v}^{v}=x_{v}^{v}
$$

from Definition 14. Because (31) accords in Definition 14, the components of a tensor of rank zero are equivalent with components of a tensor satisfying Binary law of rank zero. I rewrite (31) by consideration of $x_{\mu}^{\mu} \rightarrow x_{\mu}^{; \mu}, x_{v}^{v} \rightarrow x_{v}^{; v},(6)$, $\mu-v$ inversion form of (6) and get

$$
\begin{aligned}
\left(\frac{\partial x_{\mu}}{\partial x_{\mu}}-x_{v} \frac{1}{2}\left(\frac{\partial g^{v \mu}}{\partial x^{\mu}}\right)\right) & =\frac{\partial x^{\mu}}{\partial x^{v}} \frac{\partial x^{v}}{\partial x^{\mu}}\left(\frac{\partial x_{v}}{\partial x_{v}}-x_{\mu} \frac{1}{2}\left(\frac{\partial g^{\mu v}}{\partial x^{v}}\right)\right) \\
& =\left(\frac{\partial x_{v}}{\partial x_{v}}-x_{\mu} \frac{1}{2}\left(\frac{\partial g^{\mu v}}{\partial x^{v}}\right)\right) .
\end{aligned}
$$

-End Proof-

Proposition 9 When all coordinate systems satisfy Binary Law, $x^{\mu}=\frac{\partial x^{\mu}}{\partial x^{\nu}} x^{\nu}$ is established for $x^{\mu}$ contravariant components of a tensor satisfying Binary law of the first rank.

Proof: When all coordinate systems satisfy Binary Law, I get

$$
x^{\mu}=\frac{\partial x^{\mu}}{\partial x^{v}} x^{v}
$$

from Definition 15. Because (33) accords in Definition 15, the contravariant components of a tensor of the first rank are equivalent with contravariant components of a tensor satisfying Binary law of the first rank.

-End Proof-

Proposition 10 When all coordinate systems satisfy Binary Law, $x_{\mu}=\frac{\partial x^{\nu}}{\partial x^{\mu}} x_{v}$ is established for $x_{\mu}$ covariant components of a tensor satisfying Binary law of the first rank.

Proof: When all coordinate systems satisfy Binary Law, I get

$$
x_{\mu}=\frac{\partial x^{v}}{\partial x^{\mu}} x_{v}
$$

from Definition 16. Because (34) accords in Definition 16, the covariant components of a tensor of the first rank are equivalent with covariant components of a tensor satisfying Binary law of the first rank.

-End Proof-

Proposition 11 When all coordinate systems satisfy Binary Law, $x^{\mu \nu}=\frac{\partial x^{\mu}}{\partial x^{v}} \frac{\partial x^{v}}{\partial x^{\mu}} x^{\nu \mu}=x^{\nu \mu}$ is established for $x^{\mu \nu}$ contravariant components of a tensor satisfying Binary law of the second rank.

Proof: When all coordinate systems satisfy Binary Law, I get 


$$
x^{\mu v}=\frac{\partial x^{\mu}}{\partial x^{v}} \frac{\partial x^{v}}{\partial x^{v}} x^{v v}=\frac{\partial x^{\mu}}{\partial x^{v}} x^{v v}
$$

from Definition 17. $x^{\mu \nu}$ isn't contravariant components of a tensor satisfying Binary law of the second rank than (35). This is a problem. The dummy index has an invariable property for consideration of Binary Law. In other words, the index which was dummy index in Definition 17 is dummy index in (35). Therefore, I rewrite dummy index $v$ in $\frac{\partial x^{v}}{\partial x^{\nu}} x^{v v}$ of (35) in $\mu$ and get

$$
x^{\mu \nu}=\frac{\partial x^{\mu}}{\partial x^{v}} \frac{\partial x^{v}}{\partial x^{\mu}} x^{\nu \mu}=x^{\nu \mu} .
$$

The kind of the optional dummy index is only two kinds of $v, \mu$ in consideration of Definition 6 here. A problem in (35) is solved in (36). If I assume establishment of

$$
x^{\mu \nu}=x_{\mu}^{\mu}, x^{v \mu}=x_{v}^{v} \quad(\text { False }) .
$$

I get

$$
x_{\mu}^{\mu}=\frac{\partial x^{\mu}}{\partial x^{v}} \frac{\partial x^{v}}{\partial x^{\mu}} x_{v}^{v}=x_{v}^{v} \quad(\text { False })
$$

from (36), (37). I get

$$
x_{\mu}^{\mu}=x_{\mu}^{\mu} \quad(\text { False })
$$

from (31), (38). Because (39) isn't established,

$$
x^{\mu v}=x_{\mu}^{\mu}, x^{v \mu}=x_{v}^{v}
$$

is established.

-End Proof-

Proposition 12 When all coordinate systems satisfy Binary Law, $x_{\mu \nu}=\frac{\partial x^{v}}{\partial x^{\mu}} \frac{\partial x^{\mu}}{\partial x^{v}} x_{v \mu}=x_{v \mu}$ is established for $x_{\mu \nu}$ covariant components of a tensor satisfying Binary law of the second rank.

Proof: When all coordinate systems satisfy Binary Law, I get

$$
x_{\mu v}=\frac{\partial x^{v}}{\partial x^{\mu}} \frac{\partial x^{v}}{\partial x^{v}} x_{v v}=\frac{\partial x^{v}}{\partial x^{\mu}} x_{v v}
$$

from Definition 18. $x_{\mu v}$ isn't covariant components of a tensor satisfying Binary law of the second rank than (41). This is a problem. The dummy index has an invariable property for consideration of Binary Law. In other words, the index which was dummy index in Definition 18 is dummy index in (41). Therefore, I rewrite dummy index $v$ in $\frac{\partial x^{v}}{\partial x^{v}} x_{v v}$ of (41) in $\mu$ and get

$$
x_{\mu v}=\frac{\partial x^{v}}{\partial x^{\mu}} \frac{\partial x^{\mu}}{\partial x^{v}} x_{v \mu}=x_{v \mu} .
$$

The kind of the optional dummy index is only two kinds of $v, \mu$ in consideration of Definition 6 here. A problem in (41) is solved in (42). If I assume estab- 
lishment of

$$
x_{\mu v}=x_{v}^{v}, x_{v \mu}=x_{\mu}^{\mu} \quad(\text { False }) .
$$

I get

$$
x_{v}^{v}=\frac{\partial x^{v}}{\partial x^{\mu}} \frac{\partial x^{\mu}}{\partial x^{v}} x_{\mu}^{\mu}=x_{\mu}^{\mu} \quad(\text { False })
$$

from (42), (43). I get

$$
\left.x_{\mu}^{\mu}=x_{\mu}^{\mu} \quad \text { (False }\right)
$$

from (31), (44). Because (45) isn’t established,

$$
x_{\mu v}=x_{v}^{v}, x_{v \mu}=x_{\mu}^{\mu}
$$

is established. I rewrite (42) by consideration of $x_{\mu v} \rightarrow x_{\mu ; \nu}, x_{v \mu} \rightarrow x_{v ; \mu}$, (9), $\mu-v$ inversion form of (9) and get

$$
\left(\frac{\partial x_{\mu}}{\partial x^{v}}\right)=\frac{\partial x^{v}}{\partial x^{\mu}} \frac{\partial x^{\mu}}{\partial x^{v}}\left(\frac{\partial x_{v}}{\partial x^{\mu}}\right)=\left(\frac{\partial x_{v}}{\partial x^{\mu}}\right) .
$$

I rewrite (46) by consideration of $x_{\mu v} \rightarrow x_{\mu ; \nu}, x_{v}^{v} \rightarrow x_{v}^{; v},(9), \mu-v$ inversion form of (6) and get

$$
\frac{\partial x_{\mu}}{\partial x^{v}}=\left(\frac{\partial x_{v}}{\partial x_{v}}-x_{\mu} \frac{1}{2}\left(\frac{\partial g^{\mu v}}{\partial x^{v}}\right)\right)
$$

-End Proof-

Proposition 13 When all coordinate systems satisfy Binary Law, $x_{v}^{\mu}=\frac{\partial x^{\mu}}{\partial x^{\nu}} \frac{\partial x^{\mu}}{\partial x^{\nu}} x_{\mu}^{\nu}$ is established for $x_{v}^{\mu}$ components of the mixed tensor satisfying Binary law of the second rank.

Proof: When all coordinate systems satisfy Binary Law, I get

$$
x_{v}^{\mu}=\frac{\partial x^{\mu}}{\partial x^{v}} \frac{\partial x^{v}}{\partial x^{v}} x_{v}^{v}=\frac{\partial x^{\mu}}{\partial x^{v}} x_{v}^{v}
$$

from Definition 19. $x_{v}^{\mu}$ isn't components of the mixed tensor satisfying Binary law of the second rank than (49). This is a problem. The dummy index has an invariable property for consideration of Binary Law. In other words, the index which was dummy index in Definition 19 is dummy index in (49). Therefore, I rewrite dummy index $v$ in $\frac{\partial x^{v}}{\partial x^{v}} x_{v}^{v}$ of (49) in $\mu$ and get

$$
x_{v}^{\mu}=\frac{\partial x^{\mu}}{\partial x^{v}} \frac{\partial x^{\mu}}{\partial x^{v}} x_{\mu}^{v} .
$$

The kind of the optional dummy index is only two kinds of $v, \mu$ in consideration of Definition 6 here. A problem in (49) is solved in (50). I rewrite (50) by consideration of $x_{v}^{\mu} \rightarrow x_{;}^{\mu}, x_{\mu}^{v} \rightarrow x_{; \mu}^{v}$, (16), $\mu-v$ inversion form of (16) and get

$$
\left(\frac{\partial x^{\mu}}{\partial x^{v}}\right)=\frac{\partial x^{\mu}}{\partial x^{\nu}} \frac{\partial x^{\mu}}{\partial x^{\nu}}\left(\frac{\partial x^{v}}{\partial x^{\mu}}\right)
$$


-End Proof-

Proposition 14 When all coordinate systems satisfy Binary Law, $x_{v V}^{\mu}=\frac{\partial x^{\mu}}{\partial x^{v}} \frac{\partial x^{v}}{\partial x^{\mu}} \frac{\partial x^{\nu}}{\partial x^{\mu}} x_{\mu \mu}^{v}=\frac{\partial x^{\nu}}{\partial x^{\mu}} x_{\mu \mu}^{\nu}$ is established for $x_{v v}^{\mu}$ components of the mixed tensor satisfying Binary law of the third rank of the second rank covariant in the first rank contravariant.

Proof: When all coordinate systems satisfy Binary Law, I get

$$
x_{v v}^{\mu}=\frac{\partial x^{\mu}}{\partial x^{v}} \frac{\partial x^{v}}{\partial x^{v}} \frac{\partial x^{v}}{\partial x^{v}} x_{v v}^{v}=\frac{\partial x^{\mu}}{\partial x^{v}} x_{v v}^{v}
$$

from Definition 20. $x_{v v}^{\mu}$ isn't components of the mixed tensor satisfying Binary law of the third rank of the second rank covariant in the first rank contravariant than (52). This is a problem. The dummy index has an invariable property for consideration of Binary Law.

In other words, the index which was dummy index in Definition 20 is dummy index in (52). Therefore, I rewrite dummy index $v$ in $\frac{\partial x^{v}}{\partial x^{v}} \frac{\partial x^{v}}{\partial x^{v}} x_{v v}^{v}$ of (52) in $\mu$ and get

$$
x_{v v}^{\mu}=\frac{\partial x^{\mu}}{\partial x^{v}} \frac{\partial x^{\mu}}{\partial x^{v}} \frac{\partial x^{\mu}}{\partial x^{v}} x_{\mu \mu}^{v} .
$$

The kind of the optional dummy index is only two kinds of $v, \mu$ in consideration of Definition 6 here. A problem in (52) is solved in (53). If I assume establishment of

$$
x_{v v}^{\mu}=\frac{\partial x^{\mu}}{\partial x^{v}} \frac{\partial x^{v}}{\partial x^{\mu}} \frac{\partial x^{v}}{\partial x^{\mu}} x_{\mu \mu}^{v}=\frac{\partial x^{v}}{\partial x^{\mu}} x_{\mu \mu}^{v} \quad \text { (False) }
$$

I get

$$
\frac{\partial x^{\mu}}{\partial x^{v}} \frac{\partial x^{\mu}}{\partial x^{v}} \frac{\partial x^{\mu}}{\partial x^{v}} x_{\mu \mu}^{v}=\frac{\partial x^{\mu}}{\partial x^{v}} \frac{\partial x^{v}}{\partial x^{\mu}} \frac{\partial x^{v}}{\partial x^{\mu}} x_{\mu \mu}^{v} \quad \text { (False) }
$$

from (53), (54). I rewrite the right side of (55) using Definition 4, Definition 5 and get

$$
\begin{aligned}
\frac{\partial x^{\mu}}{\partial x^{v}} \frac{\partial x^{\mu}}{\partial x^{v}} \frac{\partial x^{\mu}}{\partial x^{v}} x_{\mu \mu}^{v} & =\frac{\partial x^{\mu}}{\partial x^{v}} \frac{\partial\left(-x^{\mu}\right)}{\partial\left(-x^{v}\right)} \frac{\partial\left(-x^{\mu}\right)}{\partial\left(-x^{v}\right)} x_{\mu \mu}^{v} \\
& =\frac{\partial x^{\mu}}{\partial x^{v}} \frac{\partial x^{\mu}}{\partial x^{v}} \frac{\partial x^{\mu}}{\partial x^{v}} x_{\mu \mu}^{v} \quad \text { (False) }
\end{aligned}
$$

Because (56) isn't established,

$$
x_{v v}^{\mu}=\frac{\partial x^{\mu}}{\partial x^{v}} \frac{\partial x^{v}}{\partial x^{\mu}} \frac{\partial x^{v}}{\partial x^{\mu}} x_{\mu \mu}^{v}=\frac{\partial x^{v}}{\partial x^{\mu}} x_{\mu \mu}^{v}
$$

is established. If I assume establishment of

$$
x_{v v}^{\mu}=x_{\mu}, x_{\mu \mu}^{v}=x_{v} \quad(\text { False }) .
$$

I get

$$
x_{\mu}=\frac{\partial x^{v}}{\partial x^{\mu}} x_{v} \quad \text { (False) }
$$


from (57), (58). I get

$$
x_{\mu}=x_{\mu} \quad(\text { False })
$$

from (34), (59). Because (60) isn't established,

$$
x_{v v}^{\mu}=x_{\mu}, x_{\mu \mu}^{v}=x_{v}
$$

is established. I rewrite (57) by consideration of $x_{v v}^{\mu} \rightarrow x_{; v ; v}^{\mu}, x_{\mu \mu}^{v} \rightarrow x_{; \mu ; \mu}^{v}$, $\mu-v$ inversion form of (23) and get

$$
\frac{\partial^{2} x^{\mu}}{\partial x^{v} \partial x^{v}}=\frac{\partial x^{\mu}}{\partial x^{v}} \frac{\partial x^{v}}{\partial x^{\mu}} \frac{\partial x^{v}}{\partial x^{\mu}} \frac{\partial^{2} x^{v}}{\partial x^{\mu} \partial x^{\mu}}=\frac{\partial x^{v}}{\partial x^{\mu}} \frac{\partial^{2} x^{v}}{\partial x^{\mu} \partial x^{\mu}} .
$$

I rewrite (61) by consideration of $x_{v v}^{\mu} \rightarrow x_{; v ; v}^{\mu},(23)$ and get

$$
\frac{\partial^{2} x^{\mu}}{\partial x^{v} \partial x^{v}}=x_{\mu}
$$

-End Proof-

Proposition 15 When all coordinate systems satisfy Binary Law, $x_{v V v}^{\mu}=\frac{\partial x^{\mu}}{\partial x^{v}} \frac{\partial x^{\mu}}{\partial x^{v}} \frac{\partial x^{v}}{\partial x^{\mu}} \frac{\partial x^{v}}{\partial x^{\mu}} x_{\mu \mu \mu}^{v}=x_{\mu \mu \mu}^{v}$ is established for $x_{v v v}^{\mu}$ components of the mixed tensor satisfying Binary law of the fourth rank of the third rank covariant in the first rank contravariant.

Proof: When all coordinate systems satisfy Binary Law, I get

$$
x_{v v V}^{\mu}=\frac{\partial x^{\mu}}{\partial x^{v}} \frac{\partial x^{v}}{\partial x^{v}} \frac{\partial x^{v}}{\partial x^{v}} \frac{\partial x^{v}}{\partial x^{v}} x_{v v v}^{v}=\frac{\partial x^{\mu}}{\partial x^{v}} x_{v v v}^{v} .
$$

from Definition 21. $x_{v V v}^{\mu}$ isn't components of the mixed tensor satisfying Binary law of the fourth rank of the third rank covariant in the first rank contravariant than (64). This is a problem. The dummy index has an invariable property for consideration of Binary Law.

In other words, the index which was dummy index in Definition 21 is dummy index in (64). Therefore, I rewrite dummy index $v$ in $\frac{\partial x^{v}}{\partial x^{v}} \frac{\partial x^{v}}{\partial x^{v}} \frac{\partial x^{v}}{\partial x^{v}} x_{v v v}^{v}$ of (64) in $\mu$ and get

$$
x_{v v v}^{\mu}=\frac{\partial x^{\mu}}{\partial x^{v}} \frac{\partial x^{\mu}}{\partial x^{v}} \frac{\partial x^{\mu}}{\partial x^{v}} \frac{\partial x^{\mu}}{\partial x^{v}} x_{\mu \mu \mu}^{v} .
$$

The kind of the optional dummy index is only two kinds of $v, \mu$ in consideration of Definition 6 here. A problem in (64) is solved in (65). If I assume establishment of

$$
x_{v v v}^{\mu}=\frac{\partial x^{\mu}}{\partial x^{v}} \frac{\partial x^{\mu}}{\partial x^{v}} \frac{\partial x^{v}}{\partial x^{\mu}} \frac{\partial x^{\nu}}{\partial x^{\mu}} x_{\mu \mu \mu}^{v}=x_{\mu \mu \mu}^{v} \quad \text { (False) } .
$$

I get

$$
\frac{\partial x^{\mu}}{\partial x^{v}} \frac{\partial x^{\mu}}{\partial x^{\nu}} \frac{\partial x^{\mu}}{\partial x^{v}} \frac{\partial x^{\mu}}{\partial x^{\nu}} x_{\mu \mu}^{\nu}=\frac{\partial x^{\mu}}{\partial x^{v}} \frac{\partial x^{\mu}}{\partial x^{v}} \frac{\partial x^{v}}{\partial x^{\mu}} \frac{\partial x^{v}}{\partial x^{\mu}} x_{\mu \mu \mu}^{\nu} \quad \text { (False) }
$$

from (65), (66). I rewrite the right side of (67) using Definition 4, Definition 5 and get 


$$
\begin{aligned}
\frac{\partial x^{\mu}}{\partial x^{v}} \frac{\partial x^{\mu}}{\partial x^{\nu}} \frac{\partial x^{\mu}}{\partial x^{\nu}} \frac{\partial x^{\mu}}{\partial x^{\nu}} x_{\mu \mu \mu}^{v} & =\frac{\partial x^{\mu}}{\partial x^{\nu}} \frac{\partial x^{\mu}}{\partial x^{\nu}} \frac{\partial\left(-x^{\mu}\right)}{\partial\left(-x^{\nu}\right)} \frac{\partial\left(-x^{\mu}\right)}{\partial\left(-x^{\nu}\right)} x_{\mu \mu \mu}^{v} \\
& =\frac{\partial x^{\mu}}{\partial x^{\nu}} \frac{\partial x^{\mu}}{\partial x^{\nu}} \frac{\partial x^{\mu}}{\partial x^{\nu}} \frac{\partial x^{\mu}}{\partial x^{\nu}} x_{\mu \mu \mu}^{v} \quad \text { (False). }
\end{aligned}
$$

Because (68) isn't established,

$$
x_{v V v}^{\mu}=\frac{\partial x^{\mu}}{\partial x^{v}} \frac{\partial x^{\mu}}{\partial x^{v}} \frac{\partial x^{v}}{\partial x^{\mu}} \frac{\partial x^{v}}{\partial x^{\mu}} x_{\mu \mu \mu}^{v}=x_{\mu \mu \mu}^{v}
$$

is established. If I assume establishment of

$$
x_{v V v}^{\mu}=x_{\mu v}, x_{\mu \mu \mu}^{\nu}=x_{v \mu} \quad(\text { False }) .
$$

I get

$$
x_{\mu \nu}=\frac{\partial x^{\mu}}{\partial x^{v}} \frac{\partial x^{\mu}}{\partial x^{v}} \frac{\partial x^{v}}{\partial x^{\mu}} \frac{\partial x^{v}}{\partial x^{\mu}} x_{v \mu}=x_{v \mu} \quad \text { (False) }
$$

from (69), (70). I get

$$
\left.x_{\mu v}=x_{\mu v} \quad \text { (False }\right)
$$

from (42), (71). Because (72) isn't established,

$$
x_{v v v}^{\mu}=x_{\mu \nu}, x_{\mu \mu \mu}^{v}=x_{v \mu}
$$

is established. I get

$$
x_{v v v}^{\mu}=x_{\mu \nu}=x_{v}^{v}, x_{\mu \mu \mu}^{v}=x_{v \mu}=x_{\mu}^{\mu}
$$

from (46), (73). I rewrite (69) by consideration of $x_{v v V}^{\mu} \rightarrow x_{;, ; ; ; ; v}^{\mu}, x_{\mu \mu \mu}^{v} \rightarrow x_{; \mu ; \mu ; \mu}^{v}$, (30), $\mu-v$ inversion form of (30) and get

$$
\frac{\partial^{3} x^{\mu}}{\partial x^{v} \partial x^{v} \partial x^{\nu}}=\frac{\partial x^{\mu}}{\partial x^{\nu}} \frac{\partial x^{\mu}}{\partial x^{\nu}} \frac{\partial x^{\nu}}{\partial x^{\mu}} \frac{\partial x^{\nu}}{\partial x^{\mu}} \frac{\partial^{3} x^{\nu}}{\partial x^{\mu} \partial x^{\mu} \partial x^{\mu}}=\frac{\partial^{3} x^{\nu}}{\partial x^{\mu} \partial x^{\mu} \partial x^{\mu}} .
$$

I rewrite (74) by consideration of $x_{v v v}^{\mu} \rightarrow x_{i ; ; ; ; v}^{\mu}, x_{\mu \nu} \rightarrow x_{\mu ; \nu}, x_{v}^{v} \rightarrow x_{v}^{; v}$, (9), (30), $\mu-v$ inversion form of (6) and get

$$
\frac{\partial^{3} x^{\mu}}{\partial x^{v} \partial x^{v} \partial x^{v}}=\frac{\partial x_{\mu}}{\partial x^{v}}=\left(\frac{\partial x_{v}}{\partial x_{v}}-x_{\mu} \frac{1}{2}\left(\frac{\partial g^{\mu v}}{\partial x^{v}}\right)\right) \text {. }
$$

I get

$$
\frac{\partial^{3} x^{\mu}}{\partial x^{v} \partial x^{\nu} \partial x^{v}}=\frac{\partial x_{\mu}}{\partial x^{\nu}}=\left(\frac{\partial x_{v}}{\partial x_{v}}-x_{\mu} \frac{1}{2}\left(\frac{\partial g^{\mu \nu}}{\partial x^{\nu}}\right)\right)=M
$$

from (76), Definition 8.

-End Proof-

\section{Discussion}

\section{About Proposition 8}

Because (31) accords in Definition 14, components of a tensor of rank zero accord in components of a tensor satisfying Binary law of rank zero.

About Proposition 9 
Because (33) accords in Definition 15, contravariant components of a tensor of the first rank accord in contravariant components of a tensor satisfying Binary law of the first rank.

About Proposition 10

Because (34) accords in Definision16, covariant components of a tensor of the first rank accord in covariant components of a tensor satisfying Binary law of the first rank.

About Proposition 13

I get

$$
\begin{aligned}
& x_{[1]}^{1}=\frac{\partial x^{1}}{\partial x^{[1]}} \frac{\partial x^{1}}{\partial x^{[1]}} x_{1}^{[1]}, x_{[2]}^{1}=\frac{\partial x^{1}}{\partial x^{[2]}} \frac{\partial x^{1}}{\partial x^{[2]}} x_{1}^{[2]}, \\
& x_{[1]}^{2}=\frac{\partial x^{2}}{\partial x^{[1]}} \frac{\partial x^{2}}{\partial x^{[1]}} x_{2}^{[1]}, x_{[2]}^{2}=\frac{\partial x^{2}}{\partial x^{[2]}} \frac{\partial x^{2}}{\partial x^{[2]}} x_{2}^{[2]}
\end{aligned}
$$

from (50) if I assume a dimensional number 2.

\section{Conflicts of Interest}

The author declares no conflicts of interest regarding the publication of this paper.

\section{References}

[1] Ichidayama, K. (2017) Journal of Modern Physics, 8, 944-963. https://doi.org/10.4236/jmp.2017.86060

[2] Ichidayama, K. (2017) Journal of Modern Physics, 8, 126-132. https://doi.org/10.4236/jmp.2017.81011

[3] Dirac, P.A.M. (1975) General Theory of Relativity. John Wiley and Sons, Inc., New York.

[4] Fleisch, D. (2012) A Student's Guide to Vectors and Tensors. Cambridge University Press, Cambridge. 